\title{
In-flight testing of the injection of the LISA Pathfinder test mass into a geodesic
}
D. Bortoluzzi ${ }^{\mathrm{a}, \mathrm{b}}$,
D. Vignotto ${ }^{\mathrm{a}, *}$,
A. Zambotti ${ }^{\mathrm{a}}$,
M. Armano ${ }^{\mathrm{c}}, \mathrm{H}$ H. Audley ${ }^{\mathrm{d}}$, J. Baird ${ }^{\mathrm{e}}$

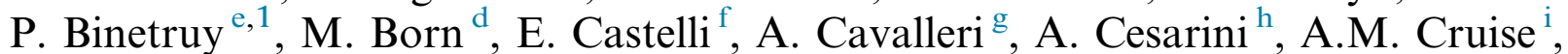
K. Danzmann ${ }^{d}$, M. de Deus Silva ${ }^{\mathrm{j}}$, I. Diepholz ${ }^{\mathrm{d}}$, G. Dixon ${ }^{\mathrm{i}}$, R. Dolesi ${ }^{\mathrm{f}}$, L. Ferraioli ${ }^{\mathrm{k}}$, V. Ferroni ${ }^{\mathrm{f}}$, E.D. Fitzsimons ${ }^{1}$, M. Freschi ${ }^{\mathrm{j}}$, L. Gesa ${ }^{\mathrm{m}, \mathrm{n}}$, F. Gibert ${ }^{\mathrm{f}, \mathrm{o}}$, D. Giardini ${ }^{\mathrm{k}}$, R. Giusteri ${ }^{\mathrm{d}}$, C. Grimani ${ }^{\text {h}}$, J. Grzymisch ${ }^{\mathrm{c}}$, I. Harrison ${ }^{\mathrm{p}}$, M.-S. Hartig ${ }^{\mathrm{d}}$, G. Heinzel ${ }^{\mathrm{d}}$, M. Hewitson ${ }^{\mathrm{d}}$, D. Hollington ${ }^{\mathrm{q}}$, D. Hoyland ${ }^{\mathrm{i}}$, M. Hueller ${ }^{\mathrm{f}}$, H. Inchauspé ${ }^{\mathrm{f}, \mathrm{r}}$, O. Jennrich ${ }^{\mathrm{c}}$, P. Jetzer $^{\mathrm{s}}$, N. Karnesis ${ }^{\mathrm{e}}$, B. Kaune ${ }^{\mathrm{d}}$, N. Korsakova ${ }^{\mathrm{u}}$, C.J. Killow ${ }^{\mathrm{t}}$, J.A. Lobo ${ }^{\mathrm{m}, \mathrm{n}, 2}$, L. Liu ${ }^{\mathrm{f}}$, J.P. López-Zaragoza ${ }^{\mathrm{m}}$, R. Maarschalkerweerd ${ }^{\mathrm{p}}{\text {, D. } \text { Mance }^{\mathrm{k}} \text {, N. Meshksar }}^{\mathrm{k}}$, V. Martín ${ }^{\mathrm{m}, \mathrm{n}}$, L. Martin-Polo ${ }^{j}$, J. Martino ${ }^{e}$, F. Martin-Porqueras ${ }^{j}$, P.W. McNamara ${ }^{c}$, J. Mendes $^{p}$, L. Mendes $^{j}$, M. Nofrarias ${ }^{\mathrm{m}, \mathrm{n}}$, S. Paczkowski ${ }^{\mathrm{d}}$, M. Perreur-Lloyd ${ }^{\mathrm{t}}$, A. Petiteau ${ }^{\mathrm{e}}$, P. Pivato ${ }^{\mathrm{f}}$, E. Plagnol ${ }^{e}$, J. Ramos-Castro ${ }^{\mathrm{v}, \mathrm{n}}$, J. Reiche ${ }^{\mathrm{d}}$, D.I. Robertson ${ }^{\mathrm{t}}$, F. Rivas ${ }^{\mathrm{m}, \mathrm{n}}$, G. Russano ${ }^{\mathrm{f}}$, J. Slutsky ${ }^{\mathrm{w}}$, C.F. Sopuerta ${ }^{\mathrm{m}, \mathrm{n}}$, T. Sumner ${ }^{\mathrm{q}}$, D. Texier ${ }^{\mathrm{j}}$, J.I. Thorpe ${ }^{\mathrm{w}}$, D. Vetrugno ${ }^{\mathrm{f}}$, S. Vitale ${ }^{\mathrm{f}}$, G. Wanner ${ }^{\mathrm{d}}$, H. Ward ${ }^{\mathrm{t}}$, P.J. Wass ${ }^{\mathrm{q}, \mathrm{r}}$, W.J. Weber ${ }^{\mathrm{f}}$, L. Wissel ${ }^{\mathrm{d}}$,

$$
\text { A. Wittchen }{ }^{\mathrm{d}} \text {, P. Zweifel }{ }^{\mathrm{k}} \text {, Carlo Zanoni }{ }^{\mathrm{a}, \mathrm{b}}
$$

\footnotetext{
a Department of Industrial Engineering, University of Trento, Via Sommarive 9, 38123 Trento, Italy

${ }^{\mathrm{b}}$ Trento Institute of Fundamental Physics and Applications, Italian Institute of Nuclear Physics (INFN), Via Sommarive 14, 38123 Trento, Italy

${ }^{\mathrm{c}}$ European Space Technology Centre, European Space Agency Keplerlaan 1, 2200 AG Noordwijk, the Netherlands

d Albert-Einstein-Institut, Max-Planck-Institut für Gravitationsphysik und Leibniz Universität Hannover, Callinstraße 38, 30167 Hannover, Germany e APC, Univ Paris Diderot, CNRS/IN2P3, CEA/lrfu, Obs de Paris, Sorbonne Paris Cité, France

${ }^{\mathrm{f}}$ Dipartimento di Fisica, Università di Trento and Trento Institute for Fundamental Physics and Application/ INFN, 38123 Povo, Trento, Italy

${ }^{\mathrm{g}}$ Istituto di Fotonica e Nanotecnologie, CNR-Fondazione Bruno Kessler, I-38123 Povo, Trento, Italy

${ }^{\mathrm{h}}$ DISPEA, Università di Urbino "Carlo Bo", Via S. Chiara, 27, 61029 UrbinolINFN, Italy

${ }^{\mathrm{i}}$ The School of Physics and Astronomy, University of Birmingham, Birmingham, UK

${ }^{\mathrm{j}}$ European Space Astronomy Centre, European Space Agency, Villanueva de la Cañada, 28692 Madrid, Spain

${ }^{\mathrm{k}}$ Institut für Geophysik, ETH Zürich, Sonneggstrasse 5, CH-8092 Zürich, Switzerland

${ }^{1}$ The UK Astronomy Technology Centre, Royal Observatory, Edinburgh, Blackford Hill, Edinburgh EH9 3HJ, UK

${ }^{\mathrm{m}}$ Institut de Ciències de l'Espai (ICE, CSIC), Campus UAB, Carrer de Can Magrans s/n, 08193 Cerdanyola del Vallès, Spain

${ }^{\mathrm{n}}$ Institut d'Estudis Espacials de Catalunya (IEEC), Cl Gran Capità 2-4, 08034 Barcelona, Spain ${ }^{\circ}$ isardSAT SL, Marie Curie 8-14, 08042 Barcelona, Catalonia, Spain

${ }^{\mathrm{p}}$ European Space Operations Centre, European Space Agency, 64293 Darmstadt, Germany

${ }^{\mathrm{q}}$ High Energy Physics Group, Physics Department, Imperial College London, Blackett Laboratory, Prince Consort Road, London SW7 2 BW, UK

${ }^{\mathrm{r}}$ Department of Mechanical and Aerospace Engineering, MAE-A, P.O. Box 116250, University of Florida, Gainesville, FL 32611, USA

${ }^{\mathrm{s}}$ Physik Institut, Universität Zürich, Winterthurerstrasse 190, CH-8057 Zürich, Switzerland

${ }^{\mathrm{t}}$ SUPA, Institute for Gravitational Research, School of Physics and Astronomy, University of Glasgow, Glasgow G12 8QQ, UK

"Observatoire de la Côte d'Azur, Boulevard de l'Observatoire CS 34229, F 06304 Nice, France

${ }^{\vee}$ Department d'Enginyeria Electrònica, Universitat Politècnica de Catalunya, 08034 Barcelona, Spain

${ }^{\mathrm{w}}$ Gravitational Astrophysics Lab, NASA Goddard Space Flight Center, 8800 Greenbelt Road, Greenbelt, MD 20771 USA
}

\footnotetext{
* Corresponding author.

E-mail address: davide.vignotto@unitn.it (D. Vignotto).

${ }^{1}$ Deceased 30 March 2017.

2 Deceased 30 September 2012.
} 
Received 24 March 2020; received in revised form 29 August 2020; accepted 2 September 2020

Available online 19 September 2020

\begin{abstract}
LISA Pathfinder is a technology demonstrator space mission, aimed at testing key technologies for detecting gravitational waves in space. The mission is the precursor of LISA, the first space gravitational waves observatory, whose launch is scheduled for 2034. The LISA Pathfinder scientific payload includes two gravitational reference sensors (GRSs), each one containing a test mass (TM), which is the sensing body of the experiment. A mission critical task is to set each TM into a pure geodesic motion, i.e. guaranteeing an extremely low acceleration noise in the sub-Hertz frequency bandwidth. The grabbing positioning and release mechanism (GPRM), responsible for the injection of the TM into a geodesic trajectory, was widely tested on ground, with the limitations imposed by the 1-g environment. The experiments showed that the mechanism, working in its nominal conditions, is capable of releasing the TM into free-fall fulfilling the very strict constraint imposed on the TM residual velocity, in order to allow its capture on behalf of the electrostatic actuation.

However, the first in-flight releases produced unexpected residual velocity components, for both the TMs. Moreover, all the residual velocity components were greater than maximum value set by the requirements. The main suspect is that unexpected contacts took place between the TM and the surroundings bodies. As a consequence, ad hoc manual release procedures had to be adopted for the few following injections performed during the nominal mission. These procedures still resulted in non compliant TM states which were captured only after impacts. However, such procedures seem not practicable for LISA, both for the limited repeatability of the system and for the unmanageable time lag of the telemetry/telecommand signals (about $4400 \mathrm{~s}$ ). For this reason, at the end of the mission, the GPRM was deeply tested in-flight, performing a large number of releases, according to different strategies. The tests were carried out in order to understand the unexpected dynamics and limit its effects on the final injection. Some risk mitigation maneuvers have been tested aimed at minimizing the vibration of the system at the release and improving the alignment between the mechanism and the TM. However, no overall optimal release strategy to be implemented in LISA could be found, because the two GPRMs behaved differently.
\end{abstract}

(C) 2020 COSPAR. Published by Elsevier Ltd. All rights reserved.

Keywords: LISA Pathfinder; Injection into geodesic motion; Space mechanism in-flight testing; Impulse measurement

\section{Introduction}

Gravitational waves detection is an exceptional scientific and engineering challenge. Space-based detection benefits of a low frequency bandwidth which is particularly interesting for the expected sources (black holes, binary systems, etc.). The laser interferometer space antenna (LISA) mission has been approved by ESA for the L3 slot (large missions) and its launch is scheduled for 2034 (Seoane et al., 2013). A mission demonstrator of LISA, named LISA Pathfinder (LPF), was launched in December 2015 and operated until June 2017. Its goal was to test some key technologies to be implemented in LISA and to verify the feasibility of the gravitational waves measurement from space (Anza et al., 2005; McNamara et al., 2008; Armano et al., 2009). The mission was a success (Armano et al., 2016; Armano et al., 2018; Wanner, 2019), even if some complications had to be faced. On the LPF spacecraft two gravitational reference sensors (GRSs, Dolesi et al., 2003; Bortoluzzi et al., 2004; Armano et al., 2017) are integrated, each one hosting a test mass (TM), which is the sensing body of the experiment. Each TM is a cubic shaped gold-platinum alloy, precisely machined. The two TMs, during the science phase, have to be set into pure geodesic motion inside two electrode housings (EHs). The sequence of operations carried out to set the TMs into a geodesic is called injection procedure.
In science mode, the spacecraft follows the free-floating TMs without affecting their relative displacement (dragfree technology, Schleicher et al., 2018; Armano et al., 2019). The electrodes surrounding each TM are used to inject a voltage, to measure its position and attitude and to control it by means of the electrostatic actuation force. What makes the GRS so challenging a system is the fact that it is designed to provide a TM acceleration noise level of $3 \times 10^{-14} \mathrm{~m} / \mathrm{s}^{2} / \sqrt{\mathrm{Hz}}$ in the measurement bandwidth 1-30 mHz. To achieve such a low noise level, three main design strategies are adopted:

- Use a relatively heavy TM (about $2 \mathrm{~kg}$ ), thus reducing the acceleration produced by a given force noise.

- Provide large gaps between the free-floating TM and the surrounding surfaces of the electrode housing, in order to limit the effect of the major sources of force noise (out-gassing, local electric fields, ect.).

- Realize a gold-coating of the TM and any surface facing the TM, in order to limit the charge patches and the stray electric fields.

Each of the three strategies presents some drawbacks. Using a heavy TM requires to design a dedicated launch lock mechanism, exerting high forces on the TM itself, thus generating strong adhesion phenomena and making the following detachment (hereinafter called release) more 
Table 1

Requirement imposed on the TM initial state at the release to guarantee that the limited-authority electrostatic actuation force is capable of controlling ad stabilizing it. Translations and rotations are expressed relatively to the geometrical center of the $\mathrm{EH}$.

\begin{tabular}{rrl}
\hline & Value & Unit \\
\hline Translations & \pm 200 & $\mu \mathrm{m}$ \\
Rotations & \pm 2000 & $\mu \mathrm{rad}$ \\
Linear velocities & \pm 5 & $\mu \mathrm{m} \mathrm{s}^{-1}$ \\
Angular velocities & \pm 100 & $\mu \mathrm{rad} \mathrm{s}^{-1}$ \\
\hline
\end{tabular}

difficult. Adhesion is increased by the gold-coating treatment, which produces strong adhesion bonds even at low contact loads. The main drawback of having large gaps between the TM and the EH walls is that the electrostatic actuation force, which is inversely proportional to the square of the distance of the TM from the electrode surface, is limited to the $\mu \mathrm{N}$ and $\mathrm{pN}$ orders of magnitude. As a consequence, the electrostatic force is capable of controlling and stabilizing the TM in the center of the EH, avoiding impacts with the surroundings, only if the initial position/attitude and the initial linear/angular velocities respect the requirements listed in Table 1 . The injection of an extended body into a geodesic is a non-trivial task common to several gravitational measurement experiments adopting a floating test mass as the sensing body (Bell, 2008; Bortoluzzi et al., 2010a). Fulfilling such tight requirements implies the design of a special injection procedure, involving different mechanisms.

In Section 2, these mechanisms are described, focusing on the expected TM residual momentum at the release. In Section 3, the telemetry data from the first in-flight releases are shown. The release mechanism behaved differently from the expectations, therefore a dedicated campaign of tests was planned at the end of the science phase, during the extended mission phase. In Section 4, the extended mission tests are analyzed and interpreted with a model that assumes a non-ideal dynamics of the injection phase and the main findings are presented. The conclusions are presented in Section 5.

\section{The caging, grabbing, positioning and release mechanisms}

The injection procedure, due to the design choice of the GRS, requires different mechanisms that handle the TM during the various mission phases. Each mechanism performs its functions through a dedicated mechanical interface, as described in Table 2.

During the launch and the following in orbit phases, the TM is locked inside the EH by the caging and vent mechanism (CVM, Zahnd et al., 2013). It is composed of eight cylindrical fingers, protruding inside the $\mathrm{EH}$, that are parallel to the telemetry $\mathrm{z}$ axis (Figs. 1 and 2) and exert a preload force in the order of $1 \mathrm{kN}$ on the TM vertices. Releasing the TM with a state compliant with the requirements listed in Table 1 is a non-trivial task. In particular, any asymmetry of the adhesion force between the TM and the mechanisms interface on the opposite TM sides converts, at the separation, into a TM momentum, easily violating the requirements on the maximum velocities (Benedetti et al., 2006; Bortoluzzi et al., 2013a; Bortoluzzi et al., 2015). It is not possible to perform a compliant release, i.e. a release which fulfills the requirements of Table 1 , by simply retracting the eight fingers away form the TM, due to the strong adhesive bonds at the interfaces and their low repeatability and strong asymmetry. Moreover, the CVM has been designed as a oneshot mechanism, so it is unable to re-lock the TM once the fingers have been retracted.

In order to successfully release the TM, it is necessary to reduce the preload force and the extension of the interface contact areas, thus reducing the strength of the adhesive bonds down to a level at which their asymmetric contribution to the TM residual velocity remains below the requirement. The preload force reduction and the contact area reduction are made possible by the transition form the CVM to the grabbing positioning and release mechanisms (GPRM), called pass-over.

\subsection{The GPRM}

The GPRM is designed to grab, to re-position and to release into free-fall the TM inside the hosting electrode housing, for the initialization of the science phase both after the CVM pass-over and at any situation requiring a safe-mode TM configuration (Mäusli et al., 2007; Neukom et al., 2009; Köker et al., 2013). The GPRM is composed of two halves. Each half holds a cylindrical shaped plunger (Fig. 2), which is moved along the $\mathrm{z}$ axis by means of a piezo-walk actuator. The heads of the

Table 2

Functions separation design: each mechanism is designed to perform a particular function.

\begin{tabular}{llll}
\hline $\begin{array}{l}\text { Mechanism } \\
\text { name }\end{array}$ & Main function & Mechanism interface & TM interface \\
\hline CVM & $\begin{array}{l}\text { caging the TM during launch and in- } \\
\text { orbit phases } \\
\text { grabbing the caged/floating TM and } \\
\text { repositioning it in the center of the } \mathrm{EH} \\
\text { releasing the TM into free-fall }\end{array}$ & $\begin{array}{l}\text { eight cylindrical fingers } \\
\text { two coaxial pyramidal/conical } \\
\text { plungers } \\
\text { two small spherical-edged tips, } \\
\text { protruding from the plunger heads. }\end{array}$ & $\begin{array}{l}\text { TM vertices, properly machined } \\
\text { fenters of the } \mathrm{TM} z-\text { and } z+\text { faces } \\
\text { surfaces at the bottom of the TM indents. }\end{array}$ \\
\hline
\end{tabular}




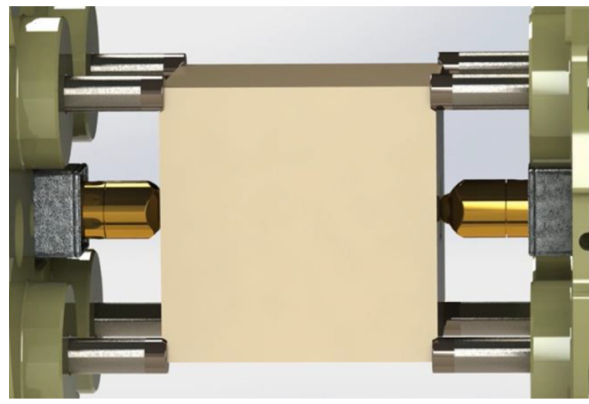

(a)

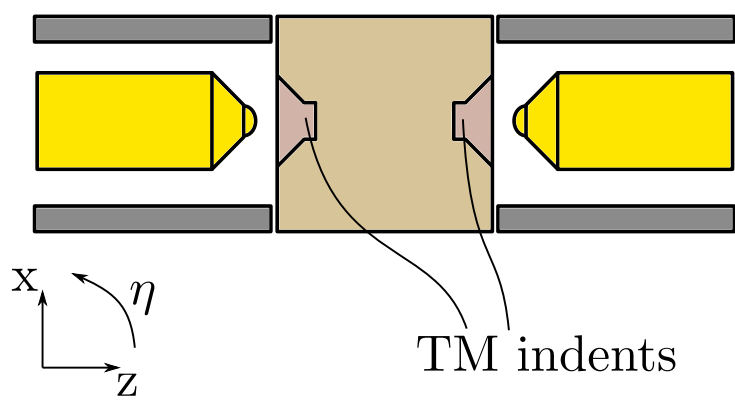

(b)

Fig. 1. On the left, rendering of the mechanisms interfaces, magnified in the locked TM configuration. The eight fingers of the CVM engage the TM on its vertices. The two plungers of the GPRM are not fully inserted into the dedicated indentations on the TM. On the right, the schematic cut-view (not to scale).

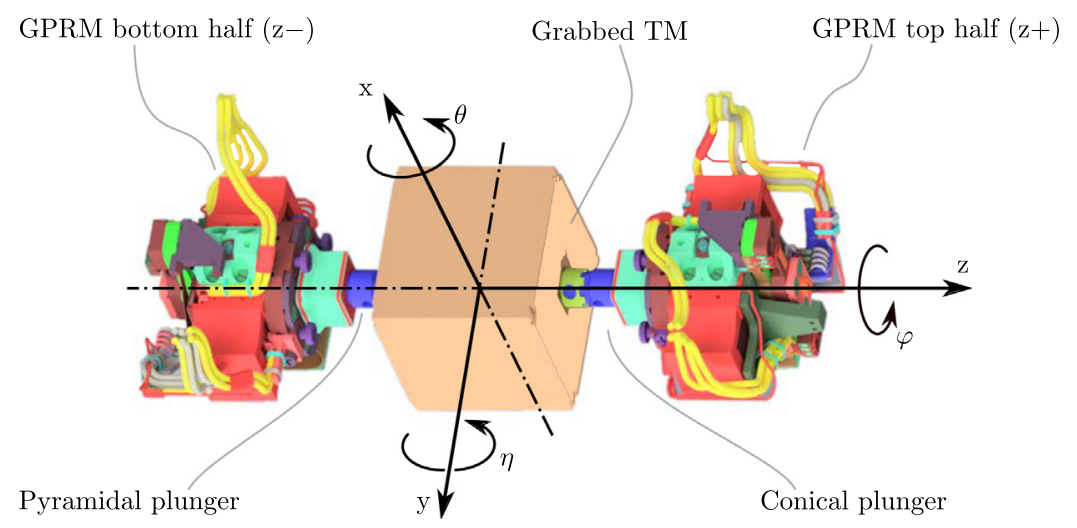

Fig. 2. GPRM $z-$ and $z+$ halves with extended plungers. TM grabbing and centering is achieved thanks to the geometry of the plunger heads and the TM indents. The reference frame of the GRS telemetry is located in the center of th EH.

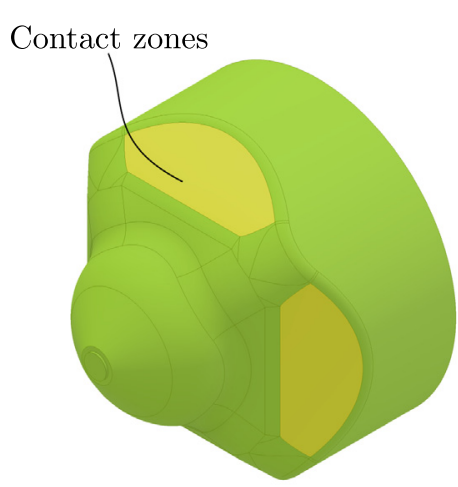

(a)

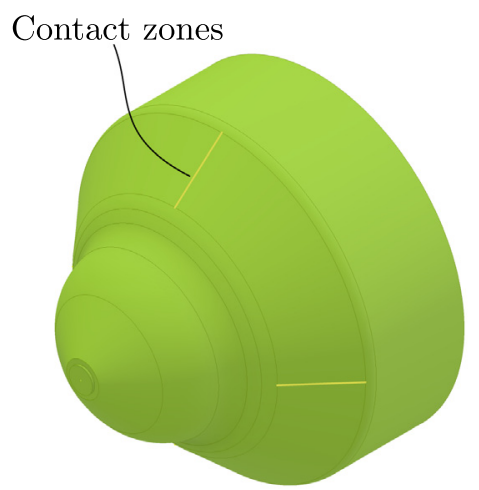

(b)

Fig. 3. Geometry detail of the plunger heads. On the left the $\mathrm{z}-$ plunger, that has a pyramidal head. On the right the ( $\mathrm{z}+$ ) plunger, that has a conical head. The yellow highlighted regions are the one where the grabbed TM touches the plunger given the nominal geometries. (For interpretation of the references to color in this figure legend, the reader is referred to the web version of this article.)

plungers are different: the one on the $\mathrm{z}-$ side has a pyramid-shaped head, the other one has a cone-shaped head (Fig. 3). Two pyramidal indents are machined in the center of the TM $z-$ and $z+$ faces, where the plunger heads come into contact with the test mass (Fig. 1). The geometries of the plunger heads and the TM indents are designed to ensure that the TM can be grabbed from any position inside its housing and automatically centered in the $\mathrm{x}-\mathrm{y}$ plane. The rotational degrees of freedom (DOFs) of the TM are also mechanically constrained by the geometries of the contact surfaces. In particular, the different shape of the top and bottom plungers is adopted not to overconstrain the TM rotation about the $\mathrm{z}$ axis (angle $\varphi$, Fig. 2). The grabbing of the TM is achieved by moving both plungers, from their retracted position, towards the center of the electrode housing using the piezo-walk 


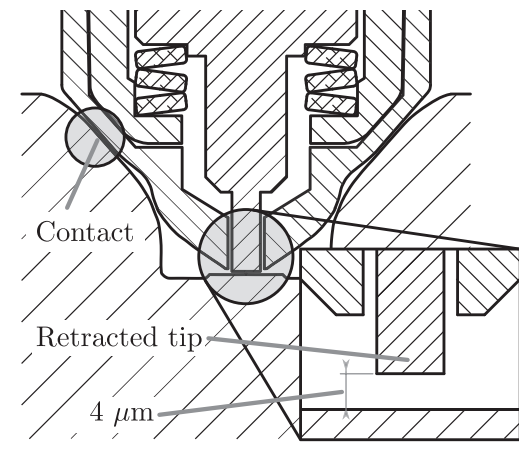

(a)

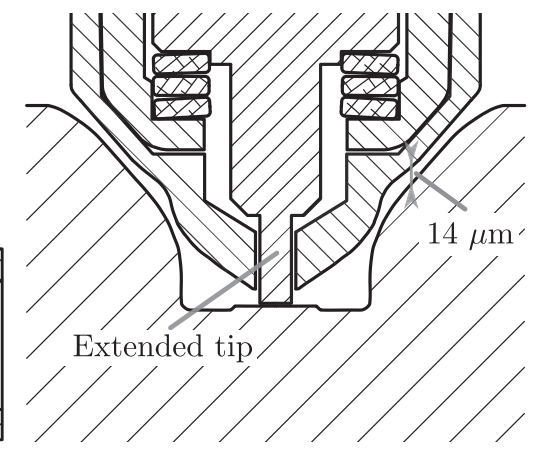

(b)

Fig. 4. (a) Cut-view of the plunger head grabbing the TM into the dedicated indent, the retracted tip free-stroke is regulated to $4 \mu \mathrm{m}$. (b) Cut-view of the handed-over TM, hold in position by the extracted tip. The vertical gap between the mass and the plunger is $14 \mu \mathrm{m}$. Drawings are not to scale.

actuator. The very first grabbing of the TM, at the passover, is followed by the retraction of the eight fingers of the CVM, which is not being used anymore. After the $\mathrm{TM}$ is grabbed, any repositioning function is carried out by commanding the piezo-walk actuators such that one plunger moves in the same direction with respect to the other along the $\mathrm{z}$ axis, ideally maintaining the TM zero attitude and zero $\mathrm{x}$ and $\mathrm{y}$ positions.

The preload force on the TM can be controlled with the piezo-walk actuators, retracting or extending the plungers. Each plunger is actuated by its piezo-walk through a cantilever structure, equipped with four strain gauges in full bridge configuration, excited by a constant voltage source. The unbalance of the Wheatstone bridge provides the measurement of the axial force on the plunger. The nominal release is performed with a preload force of $0.3 \mathrm{~N}$.

The last function of the GPRM, the release of the TM, is performed by a dedicated sub-mechanism. This system is hosted inside the plungers and is composed of two small gold-alloy cylindrical tips, coaxial with the hosting plunger, that are extended from the plunger heads by means of two piezo-stack actuators. The tip ends are spherical (with a large radius compared to the diameter), to minimize the contact surface thus reducing the adhesion force. The tip extension is provided by the expansion of the piezo-stack, produced by an applied voltage. The retraction is performed by the elastic recovery of a preloaded spring, when the voltage is shorted to zero.

The transition from the grabbed TM held in position by the plungers to the tips is called hand-over and is performed controlling the preload force on the TM. During the hand-over, in the nominal procedure, the plungers are retracted from the TM and, simultaneously, the tips are extended toward it. At the end of the hand-over, the tips are touching the TM onto two dedicated interfaces, precisely machined, called landing areas. From the specifications of the piezo stack actuator, the maximum tip stroke is $18 \mu \mathrm{m}$. The tips are regulated such that there is a gap of $4 \mu \mathrm{m}$ (free stroke gap) before touching the landing areas of the grabbed TM when extended. As a consequence, the amount of the total tip stroke that is converted into an actual retraction of the plungers from the TM during the hand-over is, at most, $18-4=14 \mu \mathrm{m}$ (see Fig. 4). This means that the TM-plunger gap at the release is very tiny if compared to the extension of the nominal contact surfaces. Given the symmetry of the system and the orientation of the GPRM, the expected motion of the TM after the nominal release is a linear motion along the $\mathrm{z}$ axis, without any rotations or $\mathrm{x}$ and $\mathrm{y}$ translations.

\subsection{GPRM on-ground testing}

Since the release of the TM is a mission critical task, dedicated experiments on the GPRM have been carried out on-ground. The goal was to test the functionality and performance of the TM release function, respecting the very strict requirement imposed on the residual velocities (Table 1). In the nominal release configuration, the TM release velocity is expected to be limited to the $\mathrm{z}$ axis and produced by the asymmetry of the contact forces between the release tips and the landing areas. In this scenario, the most unpredictable contribution to the asymmetry was identified in the adhesion force. As a consequence, at the University of Trento, the transferred momentum measurement facility (TMMF, Bortoluzzi et al., 2011a) was set up to characterize such a contribution (Benedetti et al., 2008; Bortoluzzi et al., 2008; Bortoluzzi et al., 2009; De Cecco et al., 2009; Bortoluzzi et al., 2010b; Benedetti et al., 2011; Zanoni et al., 2015; Bortoluzzi et al., 2017). The experiment was designed to estimate the momentum transferred to a suspended mock-up of the TM by the quick retraction of a single adhered tip. The TM mock-up, located inside a vacuum chamber, was fully representative (bulk material, coating, surface machining) of the actual in-flight release conditions. The mechanism used to release the TM mock-up was a qualifying model of the GPRM. Analyzing the results of the TMMF release tests, it was estimated that the GPRM, with a $0.3 \mathrm{~N}$ preload, should be able to perform a compliant release with probability $>96 \%$ (Bortoluzzi et al., 2011b; Bortoluzzi et al., 2013b; Zanoni and Bortoluzzi, 2014). 


\section{In-flight releases}

The first TM releases performed in-flight, on February 2016, to initiate the science phase of LPF, resulted in the release not being executed nominally (Bortoluzzi et al., 2016). The injections of both the TMs were performed following the steps of the designed injection procedure: the pass-over from the CVM to the plungers, followed by the hand-over to the tips, with a $0.3 \mathrm{~N}$ preload, and then the quick tip retractions (plungers are retracted simultaneously with the tips). Unexpectedly, the residual velocities of both the TMs were not compliant with the requirements. A summary of the first in-flight release velocities is presented in Table 3.

Looking at the numbers in Table 3, two criticalities must be identified, affecting both the GPRMs. First, the presence of non-zero linear and angular velocities in all the six degrees of freedom of the released TM, with the $z$ component of the linear velocity that is not predominant. Secondly, the fact that the majority of these velocities are well beyond the requirement. During the first in-flight releases, both the TMs had a high residual momentum and the actuation force was able to control and stabilize them in the center of the EH only after many undesired impacts took place, probably between the TMs and the guard-rings of the electrode housing or the plunger heads (it was necessary to maneuver manually the GPRM to reach the captured TM state).

During the nominal mission, an updated release procedure has been executed few more times ( 6 for the $\mathrm{TM}_{1}$ and 5 fo the $\mathrm{TM}_{2}$ ) after the activation of the safe mode. The updated release procedure consisted in a small retraction of the plungers, which still produced non compliant TM states and lead to impacts between the TM and the plunger heads in a confined volume around the EH center. The control was activated manually when the TM crossed the center of the housing.
The manual procedure described above cannot be applied in the LISA mission because the distance between the Earth and the spacecraft $\left(60 \times 10^{6} \mathrm{~km}\right)$ produces a telemetry and telecommand execution time lag (400 s) which precludes the possibility of in-time control. Moreover, it relies on impacts which resulted unpredictable, taking sometimes many trials and a lot of time to get to a fully captured state.

The GPRM is a key mechanism, not only for LPF, but also for LISA, because the release of the proof mass is a mission critical task. It is fundamental, for the success of LISA, to understand why it did not work accordingly to its expected behavior, because six mechanisms (12 units) will be involved in the commissioning of the LISA science phase. To understand the unexpected behavior observed in-flight, both the GPRMs have been subjected to a dedicated campaign of tests, planned during the extended mission phase. In the first part of the test campaign (29th/30th of June 2017), several releases were performed with both GPRMs in parallel, and many modifications to the nominal release procedure were implemented. During the test activities, some pre-release maneuvers, that could improve the GPRM release performance, were found:

- The hammering maneuver consists of moving one plunger back and forth by few steps $(2-3 \mu \mathrm{m})$ just before retracting the tips. This motion should improve the fit of the TM indents onto the plunger heads (Fig. 5). An improved fit should maximize the clearance between the TM and the plungers at the release.

- The slow tip retraction consists of repositioning the tips backward instead of quickly retracting them to perform the release. The tip repositioning velocity is approximatively $2.5 \mu \mathrm{m} \mathrm{s}^{-1}$ while the nominal retraction velocity is $>7 \times 10^{4} \mu \mathrm{m} \mathrm{s}^{-1}$. Using this release strategy, any preloading force should be slowly decreased in a symmetric way. Moreover, a slow tip repositioning is

Table 3

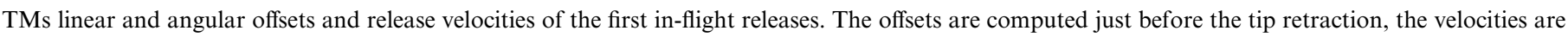

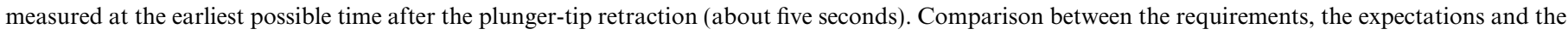
actual measurements from the firsts in-flight releases. The relative uncertainty is below $3 \%$.

\begin{tabular}{|c|c|c|c|c|c|}
\hline Unit & Component & Requirement (magnitude) & Expectation (magnitude) & $\mathrm{TM}_{1}$ & $\mathrm{TM}_{2}$ \\
\hline \multirow[t]{3}{*}{$\mu \mathrm{m}$} & $x$ & 200 & $<200$ & -0.13 & 0.02 \\
\hline & $y$ & 200 & $<200$ & -0.05 & 1.84 \\
\hline & $z$ & 200 & $<200$ & 11.04 & -0.16 \\
\hline \multirow[t]{3}{*}{$\mu \mathrm{rad}$} & $\theta$ & 2000 & $<2000$ & -17 & 50 \\
\hline & $\eta$ & 2000 & $<2000$ & -729 & 317 \\
\hline & $\varphi$ & 2000 & $<2000$ & -5 & -614 \\
\hline \multirow[t]{3}{*}{$\mu \mathrm{ms}^{-1}$} & $v_{x}$ & 5 & $\approx 0$ & -3 & +12 \\
\hline & $v_{y}$ & 5 & $\approx 0$ & -20 & -27 \\
\hline & $v_{z}$ & 5 & $<5$ & -57 & -16 \\
\hline \multirow[t]{3}{*}{$\mu \operatorname{rad~} \mathrm{s}^{-1}$} & $\omega_{\theta}$ & 100 & $\approx 0$ & +681 & +1035 \\
\hline & $\omega_{\eta}$ & 100 & $\approx 0$ & -797 & -30 \\
\hline & $\omega_{\varphi}$ & 100 & $\approx 0$ & -1085 & -430 \\
\hline
\end{tabular}



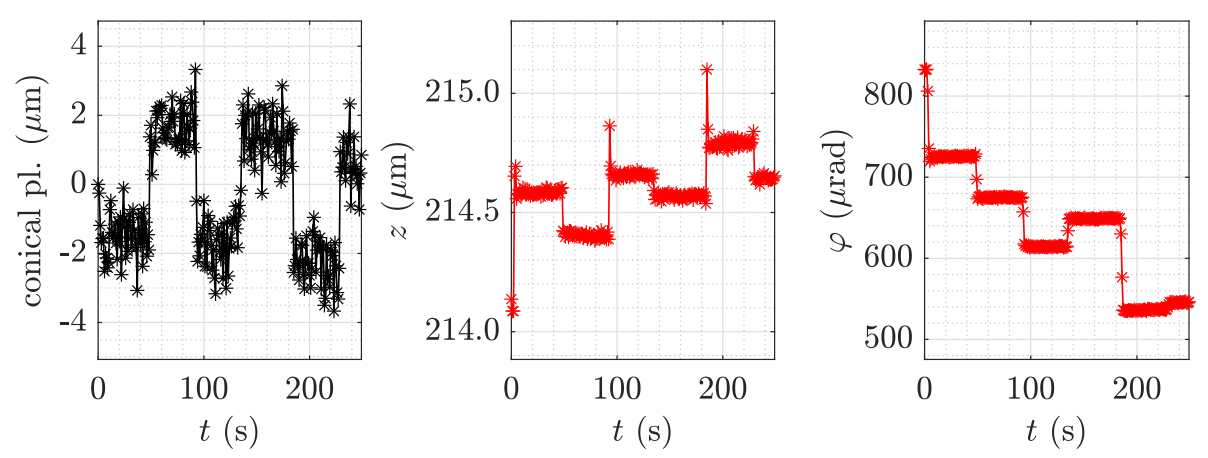

Fig. 5. Hammering maneuver on the TM: the conical plunger is moved back and forth (left graph), producing a settlement of the TM indents onto the plunger heads. As an example, $z$ (middle graph) and $\varphi$ (right graph) degrees of freedom are shown.

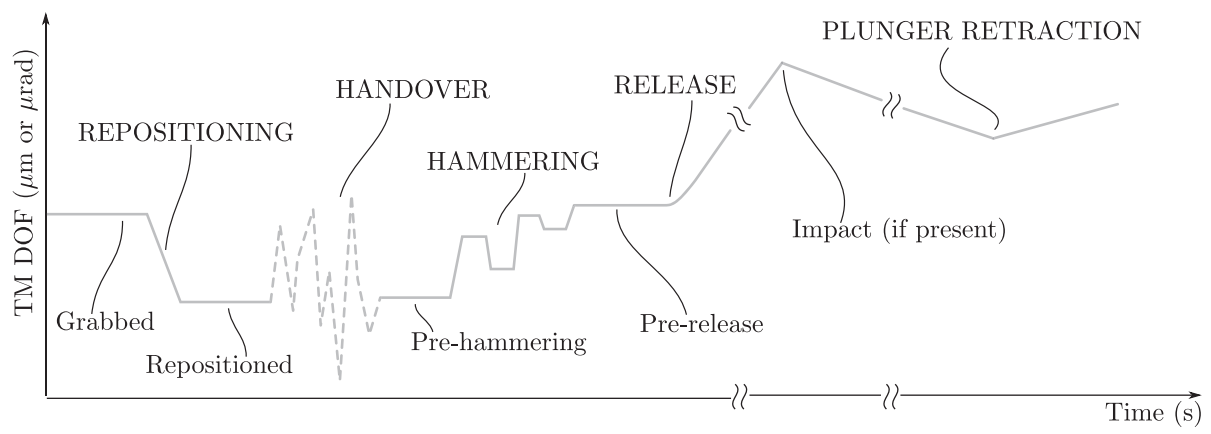

Fig. 6. Steps of an improved release procedure, from the grabbing of the TM to the plunger retraction. During the handover (from plungers to tips) the telemetry data are not available. The release can be performed by a quick or a slow tip retraction.

expected to limit the vibration of the plunger at the retraction of the tip. The drawback is not to exploit the TM inertia to break the adhesive bonds.

- The slow plunger retraction consists of moving the plungers backward, few seconds after the tips retraction, with an initial velocity of approximately $20 \mu \mathrm{m} \mathrm{s}^{-1}$, instead of $100 \mu \mathrm{m} \mathrm{s}^{-1}$ of the nominal procedure. The slower retraction of the plungers should guarantee lower oscillations, reducing the probability of a TM-tip/ plunger recontact after the release.

In Fig. 6 all the phases of the updated release procedure are shown. On the 12th/13th of July 2017, another set of tests was performed. These tests were carried out implementing an automated procedure, adopting the release procedure improvements described above. Specifically, both the GPRMs were programmed to release the TMs using two release strategies. The first is the hammering maneuver combined with a (nominal) fast tip retraction and the second is the hammering maneuver combined with a slow tip retraction. In the automated tests, the desired preload force was set to $0.5 \mathrm{~N}$ (but it was not reached in all the tests) and the plungers were slowly retracted after the release. The electrostatic actuation force was activated after the plungers withdraw had started.

The preliminary results of the automated releases are presented in Table 4. The percentages relative to the release compliance are only indicative (the estimation of the release velocities in some tests is affected by large uncer- tainty). Both the improved release strategies have worked quite well for the TM1, which was successfully captured, i.e. controlled by the electrostatic actuation force, in $91 \%$ of the fast tip tests and $100 \%$ of the slow tip tests. The second GPRM behaved worse, the TM2 was captured $78 \%$ of the times in the fast tip tests and $43 \%$ of the times in the slow tip tests. In some case, even a non-compliant release could be captured (see TM2 fast tip), thanks to the presence of some margins in the requirements on the TM initial state and/or of impacts that decreased the TM velocity dissipating its kinetic energy. On the other hand, some of the compliant releases could not be captured by the control system (see TM2 slow tip).

Despite of having adopted the improved release strategies, both the TMs were released with linear and rotational velocities different from zero, and in general the velocity along $\mathrm{z}$ axis was not the main component of the TM linear velocity.

During the extended mission phase a total of 190 tests ( 95 for each TM, in parallel) were performed, considering the slow and fast tip releases of June and July. Those tests are subdivided in 61 fast tip retractions and 34 slow tip retractions for each $\mathrm{TM}$.

When the TM is released by the GPRM, the electrostatic actuation is activated in order to control it to the nominal $\mathrm{EH}$ center. This is a non-trivial task as an unstable parasitic coupling between the TM and the spacecraft exists due to the gradients of the local electric and gravitational 
Table 4

Results of the automated releases performed in July 2017 during the extended mission phase. A release is compliant if the TM velocities immediately after the tip retraction are to within the requirements (see Table 3). The TM is considered successfully captured even after impacts.

\begin{tabular}{lcccc}
\hline & \multicolumn{2}{c}{ Fast tip } & \multicolumn{2}{c}{ Slow tip } \\
\cline { 2 - 4 } & Compliant release & Captured TM & Compliant release & Captured TM \\
\hline $\mathrm{TM}_{1}$ & $77 \%$ & $91 \%$ & $100 \%$ & \\
$\mathrm{TM}_{2}$ & $0 \%$ & $78 \%$ & $87 \%$ & $100 \%$ \\
\hline
\end{tabular}

fields. The control scheme for the TM capturing implements a non-linear sliding mode approach (Schleicher et al., 2018). According to the nominal procedure, the control system was activated when the TM state was close to the envelope defined by the requirements of Table 1 . The controller was able to stabilize and control the TM to its nominal position and attitude, if the velocities and the related linear and angular overshoots were not excessive.

\section{Analysis of the TM-plunger interactions}

In this section the in-flight data are analyzed, presenting a motivation of the unexpected release velocities. The telemetry data and other physical quantities used in this section are listed in Tables 5 and 6 (Appendix A).

The first step of the analysis is the estimation of the TM release velocity, i.e. the velocity it assumes just after the release event and defined as initial velocity. Estimating the initial velocity for any tests is non-trivial, mainly for two reasons: the presence of the signal noise and the relatively low sampling frequency $(10 \mathrm{~Hz})$ with respect to the time duration of possible impacts between the TM and the surroundings bodies. The low sampling frequency also limits the possibility to disentangle the possible contribution of a time lag in the retraction of the two tips, as few tens of microseconds are enough to convert the nominal contact preload into the maximum allowed momentum.

In some cases, it is not possible to estimate the initial velocity with a reasonable accuracy, because impacts took place just few tenths of a second after the tip retraction. In these cases it is not possible to find at least three sampled points in the TM position and attitude signals between the release and the first impact to apply a linear fit and estimate the initial velocity (see Figs. 7 and 8).

A dedicated algorithm is developed to obtain a reliable estimation of the initial velocity, and is applied to all the tests performed in flight. The main instants and phases of the injection procedure of each release are identified by comparing several telemetry signals (tips extraction, plungers positions, force sensors and TMs DOFs). The prerelease noise was estimated considering a time interval just before the TM release and computing the standard devia-
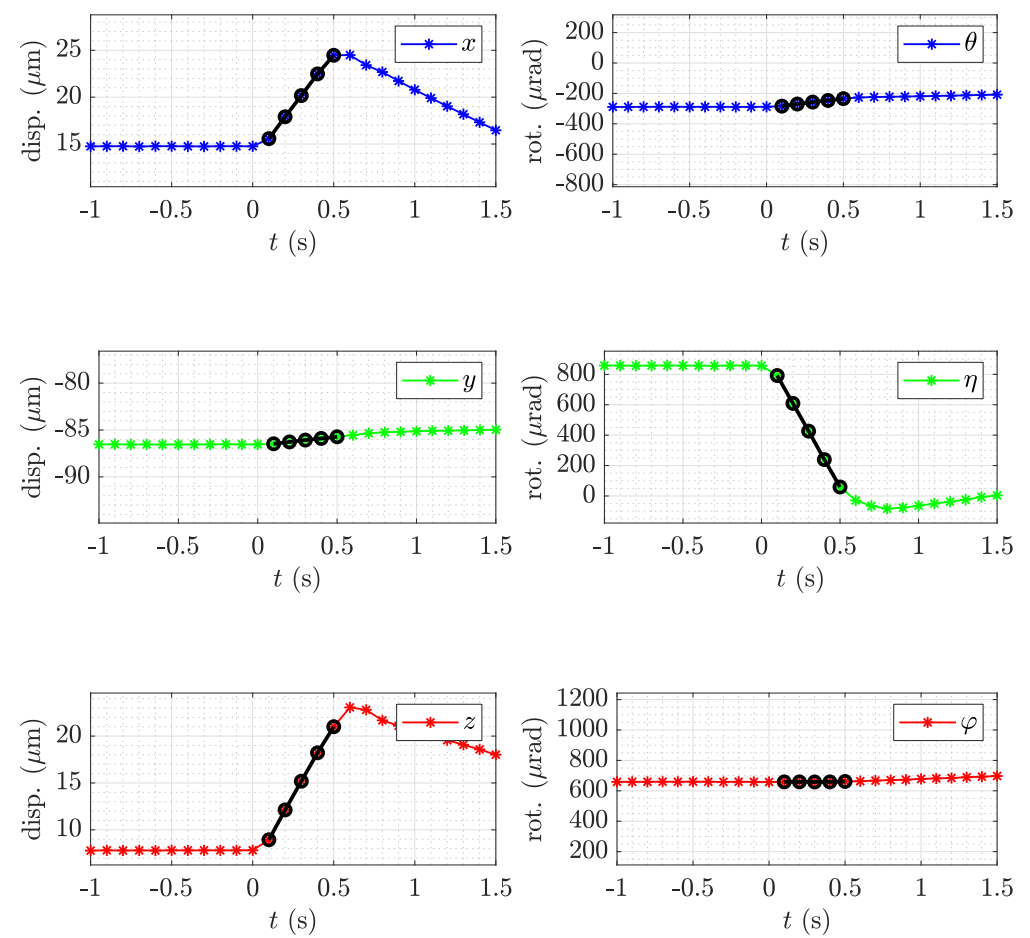

Fig. 7. Plots of the six degrees of freedom of the TM around the release instant (close to $t=0 \mathrm{~s}$ ) for a reliable test. The velocity along all degrees of freedom can be estimated with reasonable accuracy. 

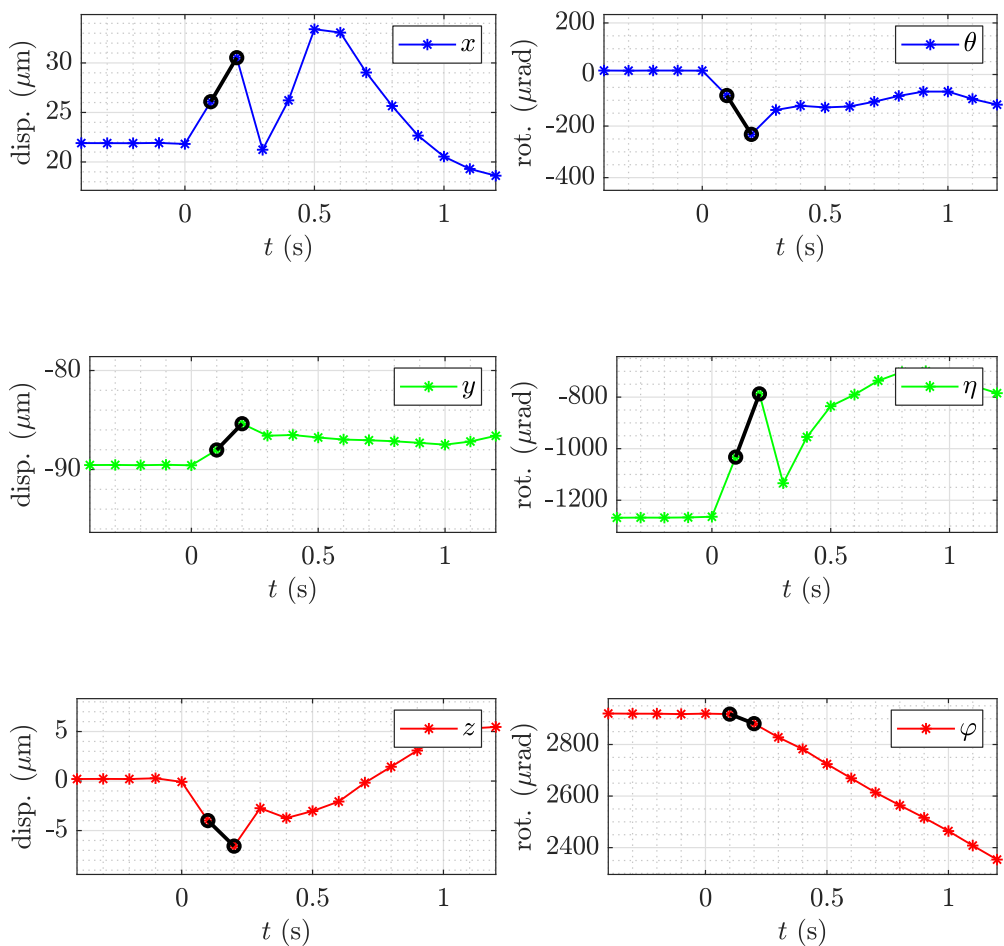

Fig. 8. Plots of the six degrees of freedom of the TM around the release instant (close to $t=0 \mathrm{~s}$ ) for a non-reliable test. It is not possible to have an accurate estimation of the release velocity along all the degrees of freedom.

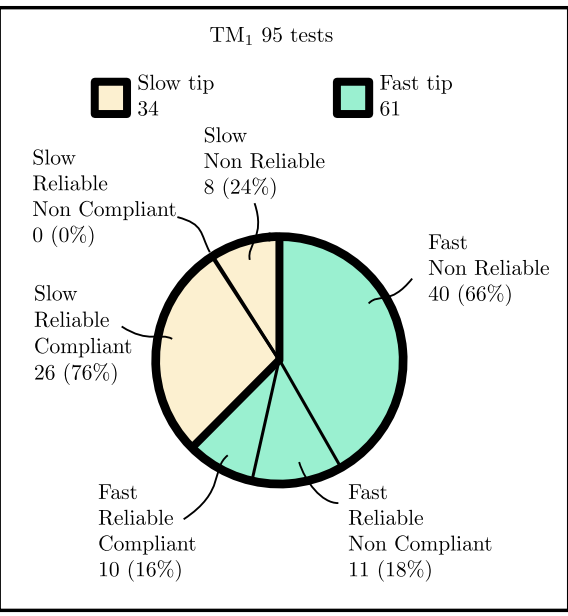

(a) $T M_{1}$

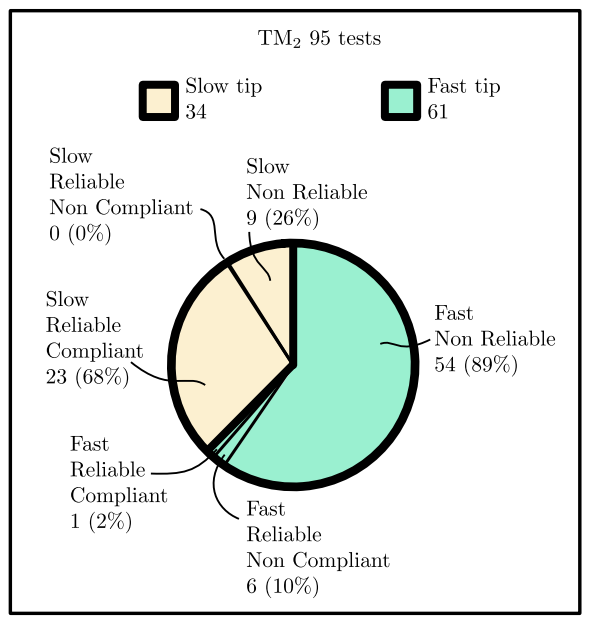

(b) $T M_{2}$

Fig. 9. Summary of the results of the algorithm applied to the tip tests performed in flight. In the picture, the sets of non-reliable tests include the tests discarded due to problems affecting the readings or the TM not being grabbed correctly before the tip retraction.

tion $\sigma_{i}$ of each TM DOF signal $(i \in\{x, y, z, \theta, \eta, \varphi\})$. After the pre-release phase, the first three consecutive points that exceeded the $\pm 3 \sigma_{i}$ interval in any of the DOFs were identified. Those points were used to estimate the initial velocity of each DOF. The initial velocity and its uncertainty were estimated applying a linear fit to the three selected points.

A possible impact in the time-frame of the three selected points is detected as a deviation from the constant velocity time history, i.e. as an event which worsen the quality of the linear fit. The direct comparison between the fit residuals and the measurement noise is not viable, because the latter is hardly characterizable in the early release conditions and a statistical test based on three samples has a limited confidence. To simplify the problem, the uncertainty of the fitted velocity (still based on the three selected points) is compared for each DOF with the respective requirement, considering that no relevant impacts are present if the absolute uncertainty is lower than $1 / 5$ of the requirement or the relative uncertainty is lower than $20 \%$. The probabil- 


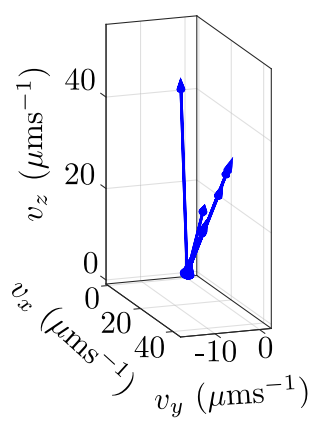

(a) $\mathrm{TM}_{1}$

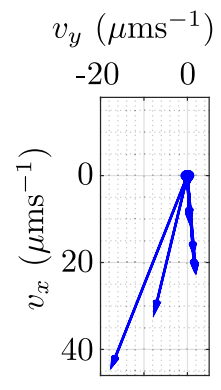

(b) $\mathrm{TM}_{1}$

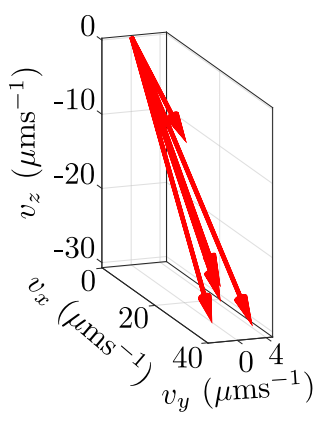

(c) $\mathrm{TM}_{2}$

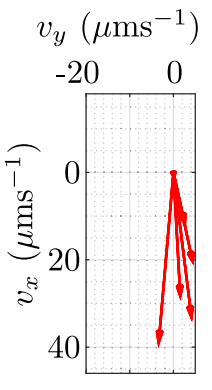

(d) $\mathrm{TM}_{2}$

Fig. 10. Linear momentum of the $\mathrm{TM}_{1}$ (blue) and $\mathrm{TM}_{2}$ (red) for the reliable non-compliant tests. The main components of the velocities of both the TMs lie on the $\mathrm{x}-\mathrm{z}$ plane. (For interpretation of the references to color in this figure legend, the reader is referred to the web version of this article.)

ity of detecting relevant impacts even by fitting only three points is enforced by the fact that those conditions need to be satisfied simultaneously by all the six DOFs signals.

If no relevant impact is detected, a test is defined as reliable. Conversely, a test is defined as non reliable if a relevant impact is detected, or if the test is affected by problems. Those problems may be a nonzero TM velocity around $\varphi$ just before the tip retraction, a very high $\varphi$ angle at the release or an occasional noise increase in the readings. The results of the application of the algorithm are summarized in Fig. 9. The reliable test detected are 47 tests of the TM1 (26 slow tip and 21 fast tip) and 30 tests of the TM2 (23 slow tip and 7 fast tip). In general, it is possible to conclude that for both TMs the slow tip strategy improves the reliability of the release. This is probably due to the fact that the slow tip retraction does not excite the vibration of the plunger. The considered sets of tests can be subdivided into compliant tests and non-compliant tests. A test is defined as compliant if all the six components of the release velocity are within the requirements. Even non-reliable tests can be subdivided applying the same criteria, with the difference that the computed release velocity has a greater uncertainty on its components. The reliable non- compliant tests ( 11 for the $\mathrm{TM}_{1}$ and 6 for the $\mathrm{TM}_{2}$ ) are studied in order to understand the main dynamics of the mechanism which is responsible for the violation of the requirements. The initial linear velocities of those tests are plotted in Fig. 10, showing that the y component of the velocity is in general much smaller than the $\mathrm{x}$ and $\mathrm{z}$ components (with few exceptions). This suggest that the release dynamics on the $\mathrm{x}-\mathrm{z}$ and $\mathrm{y}-\mathrm{z}$ planes are different.

The next step of the analysis was to estimate the impulses received by the TM at the release, to check if they are compatible with the nominal TM-tips interaction. A simple model of the TM impulses at the tip retraction was built, considering two impulses applied on the center of the landing areas, i.e. in the nominal region where the release forces should arise. The model relates the lateral components ( $\mathrm{x}$ and $\mathrm{y}$ ) of the impulses with the linear and angular velocities of the TM along $\mathrm{x}$ and $\mathrm{y}$ axes.

$$
\left\{\begin{aligned}
+l_{x}^{+}+l_{x}^{-} & =m v_{x} \\
+l_{y}^{+}+l_{y}^{-} & =m v_{y} \\
-l_{y}^{+}+l_{y}^{-} & =I_{x x} \omega_{\theta} / L \\
+l_{x}^{+}-l_{x}^{-} & =I_{y y} \omega_{\eta} / L
\end{aligned}\right.
$$

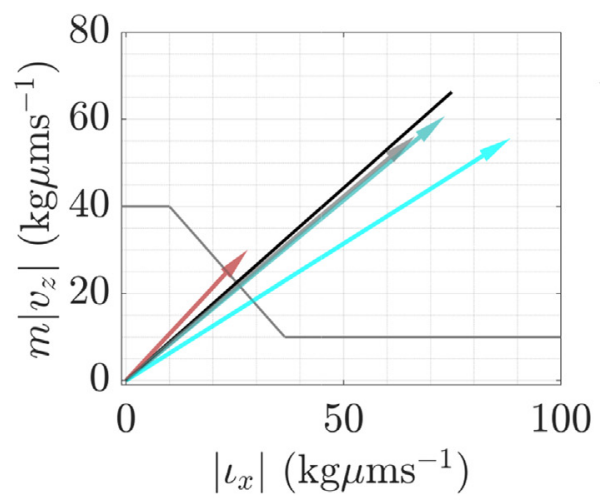

(a)

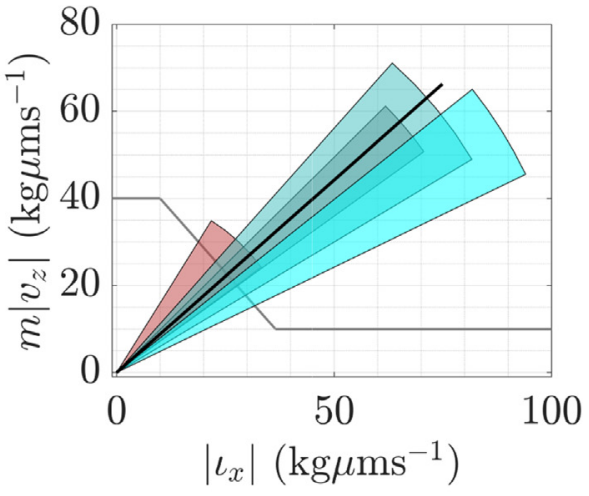

(b)

Fig. 11. Reliable, non-compliant one-sided tests with planar dynamics. Resultants of the lateral impulses computed with the simplified model and the linear momentum of the TM along z. The computed impulses (graph on the left) and the $3 \sigma$ uncertainty on the inclination (graph on the right) are shown. The black line represents the orthogonal direction with respect to the indent surface. 
Looking at the results, there are tests where the impulse on one side $(z-$ or $z+)$ is much greater than the other one (which is compatible with zero). Those tests are defined as one-sided tests. In these tests only one half of the mechanism contributed to the lateral impulse on the TM. In this case, assuming that also the $\mathrm{z}$ component of the total release impulse is generated on the same side of the mechanism, the lateral components ( $\mathrm{x}$ and $\mathrm{y}$ ) can be combined with the $\mathrm{z}$ component of the total impulse.

The TM linear momentum of the non-compliant onesided tests that had a mainly planar release dynamics $\left(l_{x} \gg l_{y}\right)$ are plotted in Fig. 11. The orthogonality of the impulses with respect to the indent surface is a strong hint of the fact that the plungers collided with the TM at the tips retraction. Conversely, it is not compatible with an interaction limited to the nominal tip-TM contact.

Thanks to this interpretation, an advanced impulse model was developed assuming that TM-plunger impacts took place at the release. The model relates the lateral impulses ( $\mathrm{x}$ and $\mathrm{y}$ directions) with the $\mathrm{TM}$ momentum along $\mathrm{z}$. This is done considering the geometry of the TM indents and imposing the orthogonality of the impulses, which was suggested by the one-sided tests (Fig. 12). In this way $r_{z}^{r e s}$, the residual impulse along $\mathrm{z}$, can be computed. It contains all the effects along $\mathrm{z}$ direction than are not explained by the projection of the lateral impulse caused by the plungers. It is difficult to estimate all the effects that caused the residual impulse $l_{z}^{\text {res }}$. In general, it includes the effects of the adhesion phenomena, the retraction delay and a $\mathrm{z}$ directed pushing effect of a plunger (Zanoni and Bortoluzzi, 2014).

$$
\begin{cases}+l_{x}^{+}+l_{x}^{-} & =m v_{x} \\ +l_{y}^{+}+l_{y}^{-} & =m v_{y} \\ \left(-l_{y}^{+}+l_{y}^{-}\right)(a+b \tan \alpha) & =I_{x x} \omega_{\theta} \\ \left(+l_{x}^{+}-l_{x}^{-}\right)(a+b \tan \alpha) & =I_{y y} \omega_{\eta} \\ \left(\left|l_{x}^{-}\right|+\left|l_{y}^{-}\right|-\left|l_{x}^{+}\right|-\left|l_{y}^{+}\right|\right) \tan \alpha+l_{z}^{\text {res }} & =m v_{z}\end{cases}
$$

The lateral impulses, computed with the advanced model, of the reliable non-compliant tests are shown in Figs. 13 and 14. From these graphs, the different behaviour of the $\mathrm{GPRM}_{1}$ and $\mathrm{GPRM}_{2}$ is clearly observable. In the GPRM releases, the $\mathrm{x}$ component of the pyramidal plunger impulse is the dominant one. In the $\mathrm{GPRM}_{2}$ releases, the $\mathrm{x}$ component of the conical plunger impulse is the dominant one.

Regarding the $\mathrm{z}$ direction, the residual impulse $l_{z}^{\text {res }}$ was computed for the same tests. Its value is lower than the momentum of the TM along $\mathrm{z}$ for all tests, even when the initial velocity is much greater than the requirement. Thus, accordingly to the model, the TM release dynamics of the reliable tests can be almost totally explained by collisions between the TM and the plungers taking place at the tip retraction. Avoiding TM-plunger collision, the mass would likely be controllable by the actuation force without further impacts, since the residual impulse (orange columns in Fig. 15) is below or very close to the requirement $\left(10 \mathrm{~kg} \mathrm{\mu m} \mathrm{s}^{-1}\right)$ most of the times (except in one case). This result is in line with the $1-\mathrm{g}$ on ground testing which concluded that the adhesion contribution, included in the residual impulse, albeit being significant, is not expected to produce excessive momentum on the TM in the majority of the cases (see Section 2.2).

The dynamical model, when applied to the non-reliable non-compliant tests, suggests the same conclusions, even if with larger uncertainty. In general, also for the non-reliable non-compliant tests, the TM dynamics is much different than the expected mono-dimensional motion along $\mathrm{z}$.

For the compliant tests, which are in most part reliable (54/55 for the TM1 and 28/35 for the TM2), the application of the dynamical model shows that the effect of the lateral impulses on the total $\mathrm{z}$ momentum is reduced, thus proving that a compliant dynamics is closer to the nominality.

\subsection{TM dynamics at the plunger retraction}

In the previous section the dynamics at the tip release has been analyzed. The tests with reliable estimation of the release velocities and high momentum (i.e. high kinetic energy) at the tip retraction (reliable non-compliant tests) have been interpreted through a model, showing that the high momentum is mainly due to the plunger-TM contacts at the indentation surfaces. This means that the TM release at the tip retraction is not nominal and the contacts between the plunger and the TM are extremely critical.

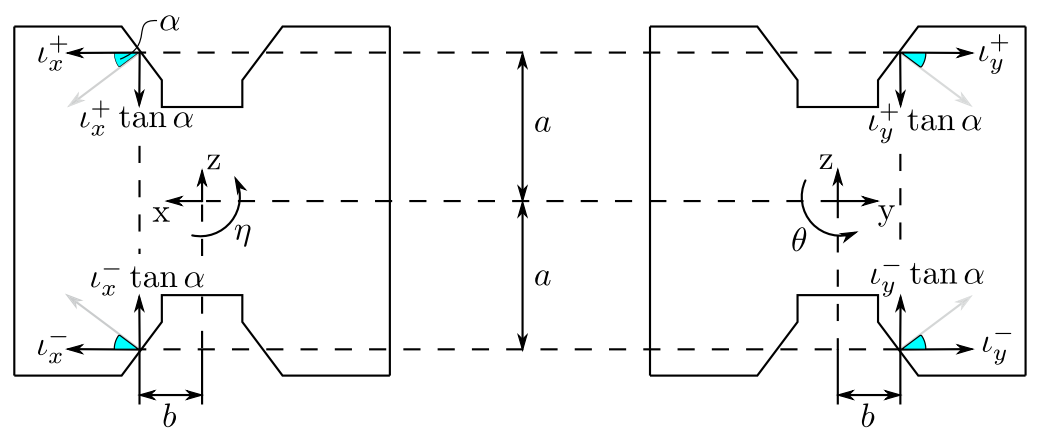

Fig. 12. Scheme of the advanced impulse model. Any lateral impulse contributes also to the TM linear momentum along z. This is done by imposing the orthogonality of each impulse to the TM indent surface. 


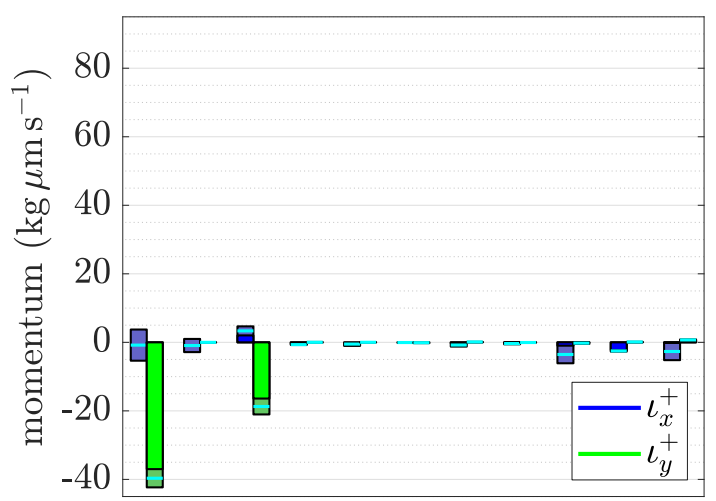

(a) $\mathrm{TM}_{1}$

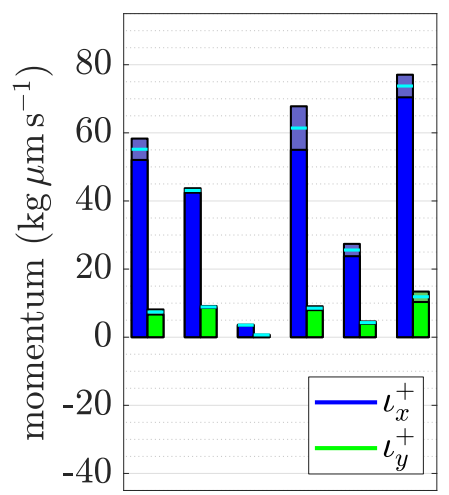

(b) $\mathrm{TM}_{2}$

Fig. 13. Reliable non-compliant tests. Comparison between the TMs release impulses on the $\mathrm{z}+$ side (conical plunger). Green columns represent the impulse in the y direction, while blue columns the impulse in the $\mathrm{x}$ direction. Each pair of columns represent a single test. For each column, the nominal value is indicated by the cyan line and the $3 \sigma$ uncertainty is represented by the colored rectangle around it. (For interpretation of the references to color in this figure legend, the reader is referred to the web version of this article.)

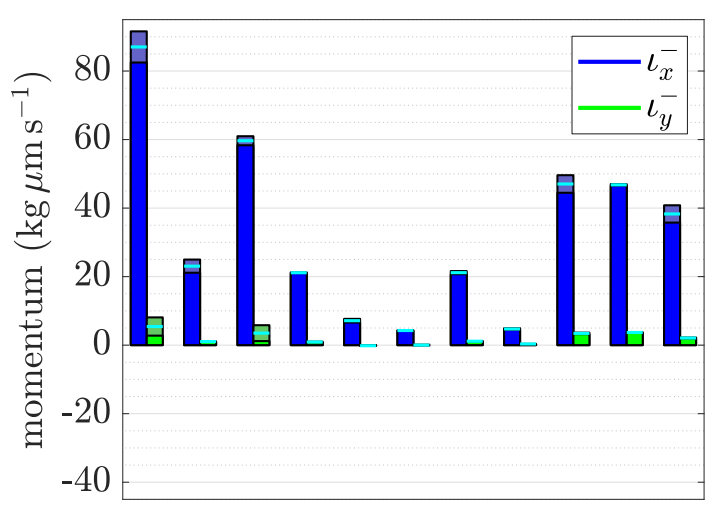

(a) $\mathrm{TM}_{1}$

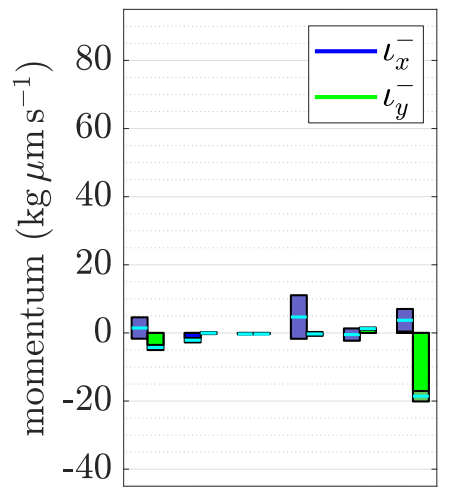

(b) $\mathrm{TM}_{2}$

Fig. 14. Reliable non-compliant tests. Comparison between the TMs release impulses on the $\mathrm{z}-$ side (pyramidal plunger). Green columns represent the impulse in the y direction, while blue columns the impulse in the $\mathrm{x}$ direction. Each pair of columns represent a single test. For each column, the nominal value is indicated by the cyan line and the $3 \sigma$ uncertainty is represented by the colored rectangle around it. (For interpretation of the references to color in this figure legend, the reader is referred to the web version of this article.)

We highlight here a second deviation from the nominal procedure: additional kinetic energy is sometimes acquired by the TM when the plungers are retracted, few seconds after the release. The hypothesis is that an additional TM-plunger contact takes place due to non-perfectly straight motion of the plunger when retracted. The dynamics of the TM-plunger interaction at the retraction of the plunger is quite more complex than the dynamics at the tip retraction. First, the actuator involved in the plunger retraction (piezo-walk) also provides the guiding function of the plunger on the $y-z$ plane, while on the $x-y$ plane a roller-slider bearing is adopted. Second, the identification of an impact cannot be performed by looking for a deviation from constant signals on the six degrees of freedom, but requires the identification of two different free-falling states. Third, a single change of the free-falling state is hardly detectable in the telemetry signals at the retraction of the plungers, whereas more impacts occur in a reduced timescale.
As a consequence, the study of the TM dynamics is limited to the kinetic energy, identifying the two constant levels which characterize its state after the tip retraction and after the plunger retraction. Even though the accuracy of the estimation is limited to the order of magnitude, some interesting comments can be done. Fig. 16 shows the relation between the kinetic energy at the tip release $\left(K_{t i p}\right)$ and the kinetic energy at the plunger retraction $\left(K_{p l}\right)$, for all the tests (independently of the reliability). For each axis, the kinetic energy is normalized with respect to the maximum compliant kinetic energy $K_{\max }$, computed from the requirements on the residual velocity listed in Table 1.

Important considerations are drawn from the graphs. The $\mathrm{TM}_{1}$ slow tip tests produce a $K_{t i p} / K_{\max }$ with maximum value around $2 \times 10^{-2}$ (cyan dots in Fig. 16(a) and (c)). When not performing the hammering, which is the case of almost any tests of Fig. 16(a), the dots are concentrated in a narrow band close to the maximum value. Performing the hammering, which is the case of almost all the tests 


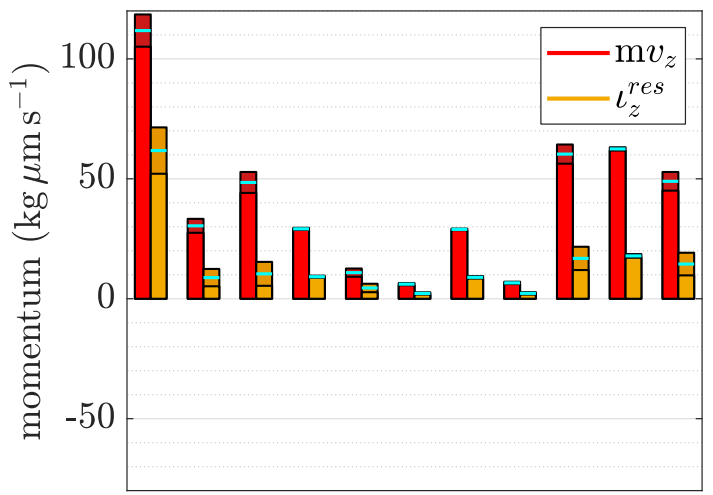

(a) $\mathrm{TM}_{1}$

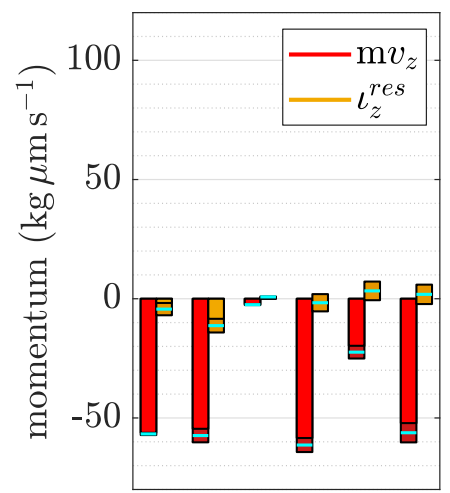

(b) $\mathrm{TM}_{2}$

Fig. 15. Reliable non-compliant tests. Comparison between the TMs linear momentum along z (red columns) and the residual impulse (orange columns) computed from the improved model of the release dynamics. Each pair of columns represent a single test. For each column, the nominal value is indicated by the cyan line and the $3 \sigma$ uncertainty is represented by the colored rectangle around it. (For interpretation of the references to color in this figure legend, the reader is referred to the web version of this article.)

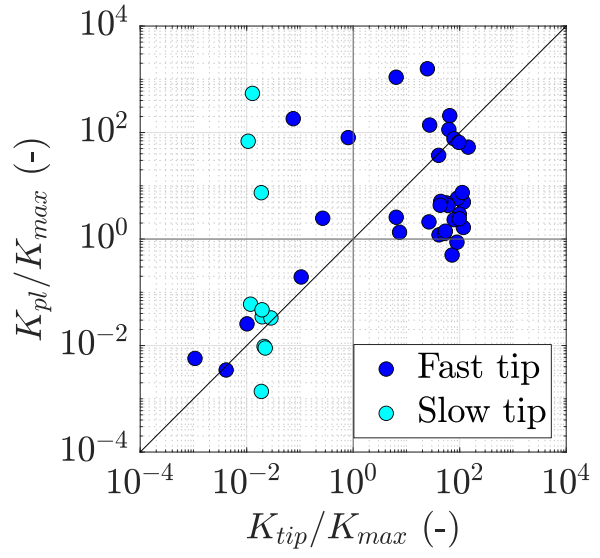

(a) $\mathrm{TM}_{1}$ Nominal plunger retraction

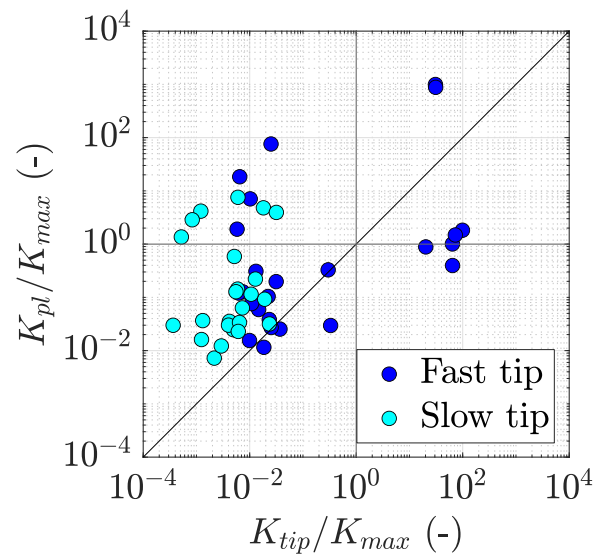

(c) $\mathrm{TM}_{1}$ Slow plunger retraction

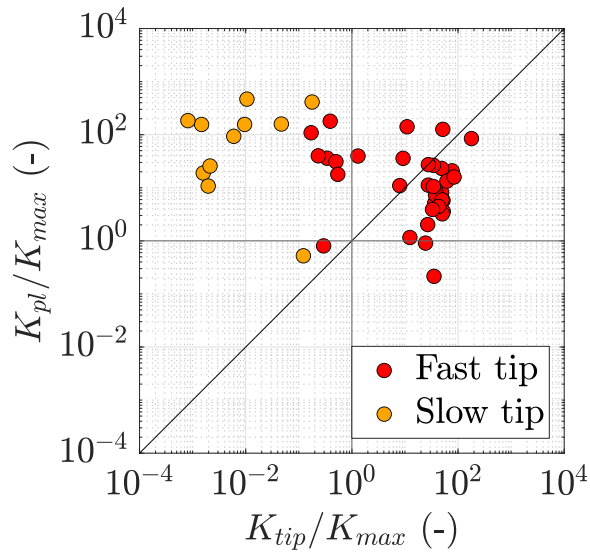

(b) $\mathrm{TM}_{2}$ Nominal plunger retraction

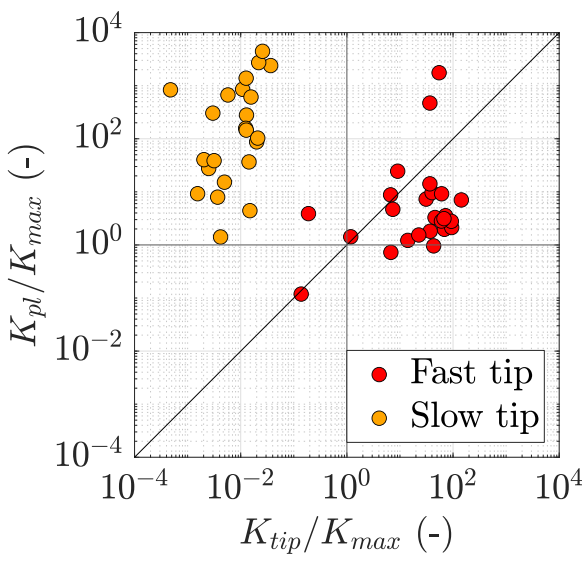

(d) $\mathrm{TM}_{2}$ Slow plunger retraction

Fig. 16. Relation between the TM kinetic energy at the tip release and the kinetic energy at the plunger retraction. Slow and fast tip tests are highlighted with different colors. Graphs (a) and (b) represent the nominal plunger retraction tests, while graphs (c) and (d) represent the slow plunger retraction tests. (For interpretation of the references to color in this figure legend, the reader is referred to the web version of this article.)

represented in Fig. 16(c), allows to reach lower values of $K_{\text {tip }}\left(10^{-4} \div 10^{-2} K_{\max }\right)$, indicating that the hammering has sometimes a positive effect on the release kinetic energy.
Based on the TM-plunger interaction model, the very low $K_{\text {tip }}$ value in the slow tip tests may be explained by the fact that the slow retraction of the tip reduces the plun- 

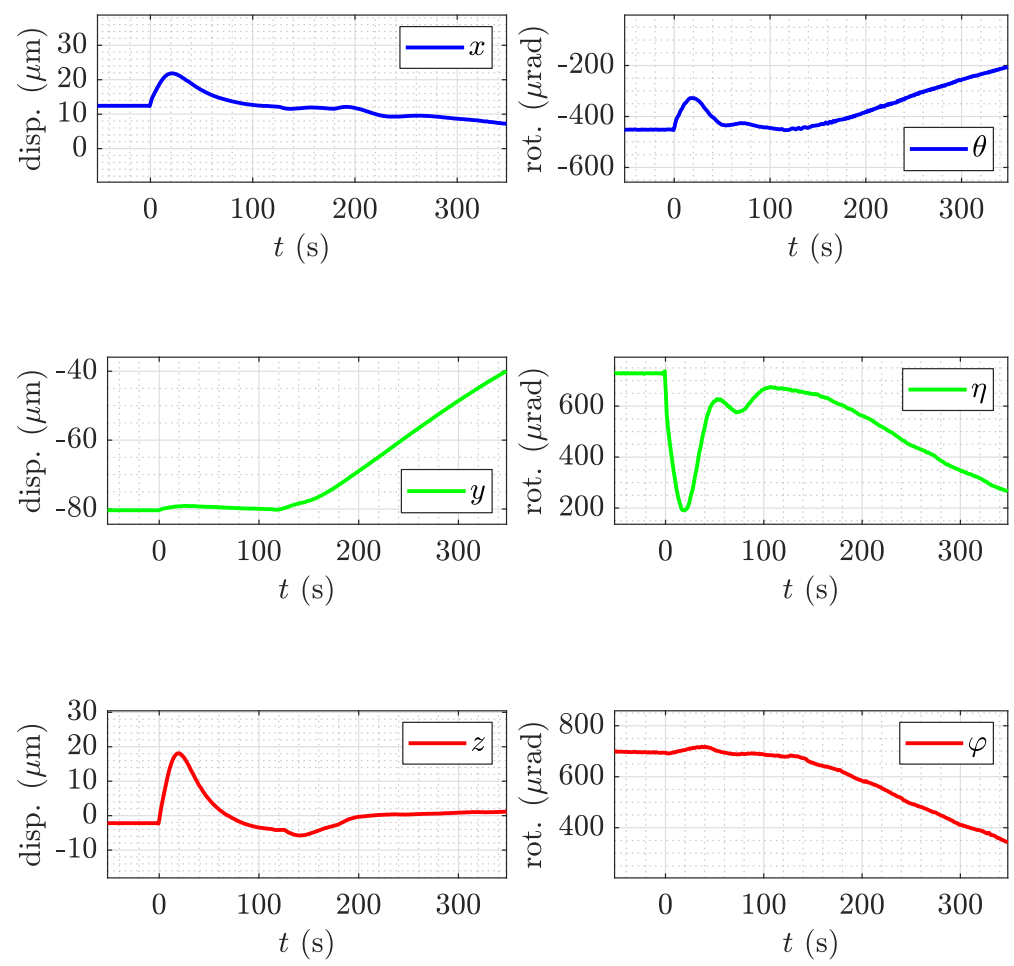

Fig. 17. Plots of the six degrees of freedom of the TM after the release instant (close to $t=0 \mathrm{~s}$ ) for the in-flight test closest to the nominal case. The test is reliable, almost compliant, with no TM-plunger impacts at the plunger retraction. The action of the control force stabilizes the TM and moves it towards the center of the EH.

ger oscillations and smoothly decreases the preload force on the TM.

Regarding the $K_{p l}$ of the slow tip tests of $\mathrm{TM}_{1}$, it is greater than $K_{\text {tip }}$ in almost any case, meaning that the TM acquires kinetic energy at the plunger retraction. When the plungers are retracted slowly (Fig. 16(c)), the $K_{p l}$ presents a lower dispersion $\left(10^{-2} \div 10^{1} K_{\max }\right)$ than when the plungers are retracted with nominal velocity $\left(10^{-3} \div 10^{3}\right.$ $K_{\max }$, Fig. 16(a)), meaning that the slow plunger retraction slightly improves the repeatability of the $K_{p} l$ and reduces the maximum kinetic energy transferred to the TM.

The values of $K_{\text {tip }} / K_{\max }$ of the fast tip retraction test of $\mathrm{TM}_{1}$ (blue dots in Fig. 16(a) and (c)) are roughly concentrated around the two values $10^{-2}$ and $10^{2}$. In particular, when the hammering maneuver is not executed, i.e. almost any test of Fig. 16(a), the blue dots are concentrated around $10^{2}$, while in Fig. 16(c) the majority of the dots are grouped around $10^{-2}$, confirming that the hammering maneuver has the positive effect of reducing the initial kinetic energy of the TM. Moreover, when $K_{t i p}$ is low, the TM tends to acquire kinetic energy at the plunger retraction, reaching high values even if plungers are retracted slowly. In contrast, when $K_{t i p}$ is high, $K_{p} l$ is in general lower than $K_{t i p}$, meaning that some dissipative phenomena take place in between the TM release and the plunger retraction, which results less critical.

Now we will focus on the $\mathrm{TM}_{2}$ tests, represented in Fig. 16(b) and (d).
The slow tip tests (orange dots) generate a $K_{\text {tip }} / K_{\max }$ spanning the range from $10^{-3}$ to $10^{-1}$. The tests of Fig. 16(b), where hammering is not performed most of the times, are more dispersed if compared to the corresponding tests of the $\mathrm{TM}_{1}$, grouped around $10^{-2}$. Executing the hammering maneuver (majority of the tests of Fig. 16(d)) still limits the dispersion of $K_{\text {tip }}\left(10^{-4} \div 10^{-2}\right.$ $K_{\max }$ ), resulting in a range similar to the $\mathrm{TM}_{1}$.

The $K_{p l}$ of slow tip tests are critical. In those tests, when the plungers are retracted, the TM always acquires a high kinetic energy, so $K_{p l}>K_{t i p}$, no matter of the plunger retraction velocity.

In particular, the dispersion of $K_{p l}$ in the nominal plunger retraction tests (Fig. 16(b)) ranges from $10^{1}$ to $10^{3}$, while in the slow plunger retraction tests (Fig. 16(d)) the dots are more dispersed and higher values of $K_{p l}$ are reached $\left(10^{0} \div 10^{4} K_{\max }\right)$. This is unexpected, since the slow plunger retraction, which improved the performance of the $\mathrm{GPRM}_{1}$, seems worsening the performance of the GPRM${ }_{2}$.

In the fast tip tests (red dots), the $\mathrm{TM}_{2}$ is released generally with a high $K_{t i p}$ (about $10^{2} K_{\max }$ ), and there are no noticeable improvements given by the hammering maneuver (Fig. 16(d)). The nominal plunger retraction tests (Fig. 16(b)) produce a $K_{p l}$ that is greater than $K_{t i p}$ when the latter is below $K_{\max }$; when $K_{\text {tip }}$ is greater that the requirement, $K_{p l}$ is in general lower than the tip release kinetic energy, meaning that some dissipative phenomena take place. 
Regarding the slow plunger retraction tests (Fig. 16(d)) the majority of the dots are under the bisector, meaning that $K_{p l}<K_{t i p}$. The are few exceptions of tests where $K_{p l}$ reaches high values, up to $10^{3} K_{\max }$, close to the $K_{p l}$ of the slow tip tests.

It is worth mentioning that, thanks to the maneuvers adopted to improve the GPRM performances, one of the automated releases of the $\mathrm{TM}_{1}$ resulted similar to the nominal case (see Fig. 17). In this test, which is reliable and compliant, the TM did not receive noticeable impacts after the tips retraction event. Moreover, the electrostatic force was able to stabilize and move the TM towards the center of the electrode-housing.

\section{Conclusions and future work}

The analysis of the flight data of the release into free-fall of the LISA Pathfinder tests masses shows that the GPRM mechanisms did not work according to their nominal design. In particular, both the TMs were initially released with unexpected velocity components and with residual velocities larger than the requirements. To understand this behavior, the GPRM $\mathrm{G}_{1}$ and $\mathrm{GPRM}_{2}$ underwent a dedicated campaign of tests at the end of the mission, during which some strategies to improve the release procedure were found (hammering, slow tip retraction, slow plunger retraction).

Analyzing the telemetry data of the TM release at the tip retraction (i.e. the actual release instant), it is found that the unexpected TM dynamics can be explained, for the reliable tests, by a concurrent TM-plunger interaction. A similar explanation can be given also for the TM dynamics at the retraction of the plungers, even though the quantitative interpretation of the measured signals is more difficult. The strategies adopted to improve the release procedure did not affect the GPRMs in the same way, meaning that no globally optimal release strategy was found. The GPRM performance strictly depends on the mechanism itself.

The slow tip retraction strategy produced very low tip release velocities for both the TMs, as expected, but for the sensor 2 worsened the TM dynamics at the plunger retraction. The hammering maneuver was effective on the $\mathrm{GPRM}_{1}$, in particular for fast tip retraction tests. The slow plunger retraction slightly improved the repeatability of the slow tip tests of the $\mathrm{GPRM}_{1}$, but worsened the performance of the same tests of the GPRM $\mathrm{M}_{2}$.

The understanding of the TM dynamics at the release, presented in this paper, represents a starting point for the improvement of the mechanism for its implementation in the forthcoming LISA mission. Six GPRM-like mechanisms will be integrated in the three LISA spacecraft, therefore their performance and repeatability constitute crucial aspects for the initialization of the science phase.

Considering the experience gained during both the nominal and the extended mission phases, no strategy is available which guarantees a successful TM injection into a geodesic with high confidence. The analysis of the reliable tests pointed out that the main problem is related to undesired TM-plunger collisions. Such interactions may be produced by either oscillations of the plunger, induced by the fast tip retraction, by the activation of the piezo-walk actuator or by the quick recovery of an elastic deformation created by the TM-plunger preload.

As a consequence, the mechanism manufacturing, assembly, integration and verification flow must be defined in order to guarantee that the release procedure is performed in-flight according to the nominal design. In particular, it seems advisable to design a TM-plunger gap budget at the release, apportioning pre-defined shares to all the contributions (static and dynamic) and providing a testbased verification of the overall compliance of the system in the flight configuration.

Future research work will concentrate on a deeper comprehension of the TM release dynamics realized in LISA Pathfinder, analyzing each contribution and identifying the relevant mechanism design features involved. The availability of a validated model of the LPF GPRM inflight release dynamics may be instrumental to the development project of the LISA GPRM.

\section{Declaration of Competing Interest}

The authors declare that they have no known competing financial interests or personal relationships that could have appeared to influence the work reported in this paper.

\section{Acknowledgements}

This work has been made possible by the LISA Pathfinder mission, which is part of the space-science programme of the European Space Agency.The French contribution has been supported by the CNES (Accord Specific de projet CNES 1316634/CNRS 103747), the CNRS, the Observatoire de Paris and the University Paris-Diderot.E. Plagnol and H. Inchauspé would also like to acknowledge the financial support of the UnivEarthS Labex program at Sorbonne Paris Cité (ANR-10-LABX-0023 and ANR-11IDEX-0005-02).The Albert-Einstein-Institut acknowledges the support of the German Space Agency, DLR. The work is supported by the Federal Ministry for Economic Affairs and Energy based on a resolution of the German Bundestag (FKZ 50OQ0501 and FKZ 50OQ1601).The Italian contribution has been supported by Agenzia Spaziale Italiana and Istituto Nazionale di Fisica Nucleare.The Spanish contribution has been supported by contracts AYA201015709 (MICINN), ESP2013-47637-P, and ESP201567234-P (MINECO).M. Nofrarias acknowledges support from Fundacion General CSIC (Programa ComFuturo). F. Rivas acknowledges an FPI contract (MINECO). The Swiss contribution acknowledges the support of the Swiss Space Office (SSO) via the PRODEX Programme of ESA. L. Ferraioli is supported by the Swiss National Science Foundation. The UK groups wish to acknowledge 
Table 5

Notation adopted to refer to the LPF telemetry signals and additional quantities defined.

\begin{tabular}{ccll}
\hline Unit & Notation & Description & Sampling frequency \\
\hline$\mu \mathrm{m}$ & $x, y, z$ & TM translations (measured) & $10 \mathrm{~Hz}$ \\
$\mu \mathrm{rad}$ & $\theta, \eta, \varphi$ & TM rotations (measured), about x, y and z axis respectively & $10 \mathrm{~Hz}$ \\
$\mathrm{~kg} \mathrm{\mu m} \mathrm{s}^{-1}$ & $l_{j}^{i}$ & impulse generated on the TM z-side $i \in\{+,-\}$ along the direction $j \in\{x, y, z\}$ & \\
$\mu \mathrm{m} \mathrm{s}^{-1}$ & $v_{j}$ & TM linear velocity component along the direction $j \in\{x, y, z\}$ & \\
$\mu \mathrm{rad} \mathrm{s}^{-1}$ & $\omega_{j}$ & TM angular velocity component around the angle $j \in\{\theta, \eta, \phi\}$ & \\
$\mathrm{pJ}$ & $K_{i}$ & TM kinetic energy at the tip or plunger retraction $(i \in\{t i p, p l\})$ & \\
\hline
\end{tabular}

Table 6

Numerical values of the parameters introduced in the analysis.

\begin{tabular}{rccl}
\hline Value & Unit & Name & Description \\
\hline 21.8 & $\mathrm{~mm}$ & $a$ & distance of the estimated TM-plunger contact point from the plane $\mathrm{x}-\mathrm{y}$ \\
3.9 & $\mathrm{~mm}$ & $b$ & distance of the estimated TM-plunger contact point from the planes $\mathrm{x}-\mathrm{z}$ or $\mathrm{y}-\mathrm{Z}$ \\
41.5 & $\circ$ & $\alpha$ & inclination of the TM indent contact surfaces with respect to the $\mathrm{z}$ axis \\
$678 \times 10^{-6}$ & $\mathrm{~kg} \mathrm{~m}^{2}$ & $I_{j j}$ & TM inertia about $j \in\{x, y\}$ axis \\
$682 \times 10^{-6}$ & $\mathrm{~kg} \mathrm{~m}^{2}$ & $I_{z z}$ & TM inertia about $z$ axis \\
\hline
\end{tabular}

support from the United Kingdom Space Agency (UKSA), the University of Glasgow, the University of Birmingham, Imperial College, and the Scottish Universities Physics Alliance (SUPA). J. I. Thorpe and J. Slutsky acknowledge the support of the US National Aeronautics and Space Administration (NASA).

\section{Appendix A. Physical quantities and parameters}

In this appendix are reported the physical quantities defined in the paper and the values of the parameters used (see Tables 5 and 6).

\section{References}

Anza, S., Armano, M., Balaguer, E., Benedetti, M., Boatella, C., Bosetti, P., Bortoluzzi, D., Brandt, N., Braxmaier, C., Caldwell, M., et al., 2005. The LTP experiment on the LISA Pathfinder mission. Class. Quantum Gravity 22 (10), S125.

Armano, M., Audley, H., Auger, G., Baird, J., Bassan, M., Binetruy, P., Born, M., Bortoluzzi, D., Brandt, N., Caleno, M., et al., 2016. Subfemto-g free fall for space-based gravitational wave observatories: LISA Pathfinder results. Phys. Rev. Lett. 116 (23), 231101.

Armano, M., Audley, H., Auger, G., Baird, J., Bassan, M., Binetruy, P., Born, M., Bortoluzzi, D., Brandt, N., Caleno, M., et al., 2017. Capacitive sensing of test mass motion with nanometer precision over millimeter-wide sensing gaps for space-borne gravitational reference sensors. Phys. Rev. D 96 (6), 062004.

Armano, M., Audley, H., Baird, J., Binetruy, P., Born, M., Bortoluzzi, D., Castelli, E., Cavalleri, A., Cesarini, A., Cruise, A.M., Danzmann, K., Silva, M., Diepholz, I., Dixon, G., Dolesi, R., Ferraioli, L., Ferroni, V., Fitzsimons, E., Freschi, M., Zweifel, P., 2018. Beyond the required LISA free-fall performance: New LISA Pathfinder results down to 20 micro-Hz. Phys. Rev. Lett. 120.

Armano, M., Audley, H., Baird, J., Binetruy, P., Born, M., Bortoluzzi, D., Castelli, E., Cavalleri, A., Cesarini, A., Cruise, A., et al., 2019. LISA Pathfinder platform stability and drag-free performance. Phys. Rev. D 99 (8), 082001.

Armano, M., Benedetti, M., Bogenstahl, J., Bortoluzzi, D., Bosetti, P., Brandt, N., Cavalleri, A., Ciani, G., Cristofolini, I., Cruise, A.M., et al., 2009. LISA Pathfinder: the experiment and the route to LISA. Class. Quantum Gravity 26 (9), 094001.

Bell, T., 2008. Hearing the heavens. Nature 452 (7183), 18-21.
Benedetti, M., Bortoluzzi, D., Baglivo, L., Vitale, S., 2011. An optimal two-input approach for impulse measurements in the nanon-s range produced by contact forces. Mech. Syst. Sig. Process. 25 (5), 1646 1660.

Benedetti, M., Bortoluzzi, D., Da Lio, M., Fontanari, V., 2006. The influence of adhesion and sub-newton pull-off forces on the release of objects in outer space. J. Tribol. Trans. ASME 128, 828-840.

Benedetti, M., Bortoluzzi, D., Vitale, S., 2008. A momentum transfer measurement technique between contacting free-falling bodies in the presence of adhesion. J. Appl. Mech. 75 (1).

Bortoluzzi, D., Armano, M., Audley, H., Auger, G., Baird, J., Binetruy, P., Born, M., Brandt, N., Bursi, A., Caleno, M., Cavalleri, A., Cesarini, A., Conklin, J.E., Cruise, M., Danzmann, K., Diepholz, I., Dolesi, R., Dunbar, N., Ferraioli, L., Ferroni, V., Fitzsimons, E., Freschi, M., Slutsky, J., Thorpe, J.I., 2016. Injection of a body into a geodesic: Lessons learnt from the LISA Pathfinder case. In: 43rd Aerospace Mechanisms Symp. (AMS).

Bortoluzzi, D., Baglivo, L., Benedetti, M., Biral, F., Bosetti, P., Cavalleri, A., Da Lio, M., De Cecco, M., Dolesi, R., Lapolla, M., et al., 2009. LISA Pathfinder test mass injection in geodesic motion: status of the on-ground testing. Class. Quantum Gravity 26 (9), 094011.

Bortoluzzi, D., Benedetti, M., Baglivo, L., De Cecco, M., Vitale, S., 2011 a. Measurement of momentum transfer due to adhesive forces: Onground testing of in-space body injection into geodesic motion. Rev. Sci. Instrum. 82 (12), 125107.

Bortoluzzi, D., Benedetti, M., Baglivo, L., Vitale, S., 2010b. A new perspective in adhesion science and technology: testing dynamic failure of adhesive junctions for space applications. Exp. Mech. 50 (8), 1213 1223.

Bortoluzzi, D., Benedetti, M., Conklin, J.W., 2013a. Indirect measurement of metallic adhesion force as a function of elongation under dynamic conditions. Mech. Syst. Sig. Process. 38 (2), 384-398.

Bortoluzzi, D., Carbone, L., Cavalleri, A., Da Lio, M., Dolesi, R., Hoyle, C., Hueller, M., Vitale, S., Weber, W., 2004. Measuring random force noise for LISA aboard the LISA Pathfinder mission. Class. Quantum Gravity 21 (5), S573.

Bortoluzzi, D., Conklin, J.W., Zanoni, C., 2013b. Prediction of the LISAPathfinder release mechanism in-flight performance. Adv. Space Res. 51 (7), 1145-1156.

Bortoluzzi, D., De Cecco, M., Vitale, S., Benedetti, M., 2008. Dynamic measurements of impulses generated by the separation of adhered bodies under near-zero gravity conditions. Exp. Mech. 48 (6), 777-787.

Bortoluzzi, D., Foulon, B., García, M.C., Lamarre, D., 2010a. Object injection in geodesic conditions: In-flight and on-ground testing issues. Adv. Space Res. 45, 1358-1379. 
Bortoluzzi, D., Mäusli, P.A., Antonello, R., Nellen, P.M., 2011 b. Modeling and identification of an electro-mechanical system: the LISA grabbing positioning and release mechanism case. Adv. Space Res. 47 (3), 453-465.

Bortoluzzi, D., Zanoni, C., Conklin, J., 2017. On-ground testing of the role of adhesion in the LISA-Pathfinder test mass injection phase. Adv. Space Res. 59 (10), 2572-2582.

Bortoluzzi, D., Zanoni, C., Vitale, S., 2015. Improvements in the measurement of metallic adhesion dynamics. Mech. Syst. Sig. Process. $52,600-613$

De Cecco, M., Bortoluzzi, D., Baglivo, L., Benedetti, M., Da Lio, M., 2009. Measurement of the momentum transferred between contacting bodies during the LISA test-mass release phase-uncertainty estimation. Meas. Sci. Technol. 20 (5), 055101.

Dolesi, R., Bortoluzzi, D., Bosetti, P., Carbone, L., Cavalleri, A., Cristofolini, I., DaLio, M., Fontana, G., Fontanari, V., Foulon, B., et al., 2003. Gravitational sensor for LISA and its technology demonstration mission. Class. Quantum Gravity 20 (10), S99.

Köker, I., Rozemeijer, H., Stary, F., Reichenberger, K., 2013. Alignment and testing of the GPRM as part of the LTP caging mechanism, in '15th Euro. In: Space Mechanisms \& Tribology Symp. (ESMATS).

Mäusli, P., Neukom, A., Romano, R., Köker, I., Durant, S., 2007. Development of a novel piezo actuated release mechanism, in '12th Euro. In: Space Mechanisms \& Tribology Symp. (ESMATS).
McNamara, P., Vitale, S., Danzmann, K., Pathfinder Science Working Team, L., et al., 2008. 'LISA pathfinder. Class. Quantum Gravity 25 (11), 114034.

Neukom, A., Romano, R., Nellen, P.M., 2009. Testing and lessons learnt of LISA GPRM, in '13th Euro. In: Space Mechanisms \& Tribology Symp. (ESMATS).

Schleicher, A., Ziegler, T., Schubert, R., Brandt, N., Bergner, P., Johann, U., Fichter, W., Grzymisch, J., 2018. In-orbit performance of the LISA Pathfinder drag-free and attitude control system. CEAS Space J. 10 (4), 471-485.

Seoane, P.A., Aoudia, S., Audley, H., Auger, G., Babak, S., Baker, J., Barausse, E., Barke, S., Bassan, M., Beckmann, V., et al., 2013. 'The gravitational universe', arXiv preprint arXiv: 1305.5720.

Wanner, G., 2019. Space-based gravitational wave detection and how LISA Pathfinder successfully paved the way. Nat. Phys. 15 (3), 200-202.

Zahnd, B., Zimmermann, M., Spörri, R., 2013. LISA Pathfinder cage and vent mechanism - development and qualification, in ' 15 th Euro. In: Space Mechanisms \& Tribology Symp. (ESMATS).

Zanoni, C., Bortoluzzi, D., 2014. Experimental-analytical qualification of a piezoelectric mechanism for a critical space application. IEEE/ ASME Trans. Mechatron. 20 (1), 427-437.

Zanoni, C., Bortoluzzi, D., Conklin, J.W., Köker, I., Seutchat, B., Vitale, S., 2015. Summary of the results of the LISA-Pathfinder test mass release. J. Phys: Conf. Ser. 610, 012022. 